Average Corotation of Line Segments Near a Point and Vortex Identification

\author{
V. Kolár̆, J. Šistek, F. Cirak and P. Moses
}

Preprint no. 2013-018 


\title{
Average Corotation of Line Segments Near a Point and Vortex Identification
}

\author{
Václav Kolár̆ \\ Institute of Hydrodynamics, Academy of Sciences of the Czech Republic, \\ Pod Patankou 30/5, CZ-16612 Prague 6, Czech Republic, \\ Phone: +420-233109095, E-mail: kolar@ih.cas.cz \\ Jakub Š́stek \\ Institute of Mathematics, Academy of Sciences of the Czech Republic, \\ Žitná 25, CZ-11567 Prague 1, Czech Republic, \\ Phone: +420-222090710, E-mail: sistek@math.cas.cz \\ Fehmi Cirak \\ Department of Engineering, University of Cambridge, \\ Trumpington Street, Cambridge CB2 1PZ, United Kingdom, \\ Phone:+44(0)1223332716, E-mail: f.cirak@eng.cam.ac.uk \\ Pavel Moses \\ Department of Mathematics, Faculty of Mechanical Engineering, \\ Czech Technical University in Prague, \\ Karlovo nám. 13, CZ-12135 Prague 2, Czech Republic, \\ Phone: +420-224357538,E-mail: moses.p@seznam.cz
}

\begin{abstract}
An easy-to-interpret kinematic quantity measuring the average corotation of material line segments near a point is introduced and applied to vortex identification. At a given point, the vector of average corotation of line segments is defined as the average of the instantaneous local rigid-body rotation over 'all planar cross-sections' passing through the examined point. The vortex identification method based on average corotation is a one-parameter, region-type local method sensitive to the axial stretching rate as well as to the inner configuration of the velocity gradient tensor. The method is derived from a well-defined interpretation of the local flow kinematics to determine the 'plane of swirling' and is also applicable to compressible and variable-density flows. Practical application to DNS data sets includes a hairpin vortex of boundary-layer transition, the reconnection process of two Burgers vortices, a flow around an inclined flat plate, and a flow around a revolving insect wing. The results agree well with some popular local methods and perform better in regions of strong shearing.
\end{abstract}




\section{Nomenclature}

\begin{tabular}{|c|c|c|}
\hline Ma & $=$ & Mach number \\
\hline $\mathbf{n}$ & $=$ & unit normal vector \\
\hline $\mathbf{n}_{\text {SWIRL }}$ & $=$ & unit normal vector of the 'plane of swirling' \\
\hline$N_{\vartheta}, N_{\varphi}$ & $=$ & $\begin{array}{l}\text { number of discretization intervals for spherical-coordinate angles } \vartheta \text { and } \varphi \\
\text { respectively }\end{array}$ \\
\hline$p_{1}, p_{2}, p_{3}$ & $=$ & strain-rate tensor principal axes \\
\hline$P$ & $=$ & arbitrary point in the flow field \\
\hline Q & $=$ & orthogonal linear transformation, $3 \times 3$ rotation matrix \\
\hline$Q$ & $=$ & second invariant of $\nabla \mathbf{u}$ defined by (A.1), vortex-identification criterion \\
\hline$r, \varphi, \vartheta$ & $=$ & spherical coordinates \\
\hline $\operatorname{Re}$ & $=$ & Reynolds number \\
\hline $\mathbf{S}$ & $=$ & strain-rate tensor, symmetric part of $\nabla \mathbf{u}$ \\
\hline$\left(s_{i j}\right)_{D}$ & $=$ & deviatoric strain-rate tensor in $2 \mathrm{D}$ \\
\hline$s_{D},\left|s_{D}\right|$ & $=$ & deviatoric principal strain-rate in $2 \mathrm{D}$ and its magnitude \\
\hline $\mathbf{u}, u_{i}$ & $=$ & velocity vector \\
\hline$u, v, w$ & $=$ & components of the velocity vector \\
\hline$V_{O}, V_{N}$ & $=$ & overlapping and non-overlapping volumes of vortex region \\
\hline $\mathrm{x}, \mathrm{x}^{\prime}$ & $=$ & position vector, the same after orthogonal transformation \\
\hline $\mathbf{x}_{0}$ & $=$ & given position vector \\
\hline$x, y, z$ & $=$ & coordinates \\
\hline$x^{\prime}, y^{\prime}, z^{\prime}$ & $=$ & new coordinates after orthogonal transformation \\
\hline$\nabla \mathbf{u}, \widehat{\nabla \mathbf{u}}$ & $=$ & velocity-gradient tensor, the same after orthogonal transformation \\
\hline$\alpha$ & $=$ & scaling factor in (12) and Appendix C \\
\hline$\delta$ & $=$ & angular step size \\
\hline$\vartheta_{i}, \varphi_{j}$ & $=$ & discretized spherical-coordinate angles $\vartheta$ and $\varphi$ \\
\hline$\Delta$ & $=$ & vortex-identification discriminant defined by (A.2) \\
\hline$\lambda_{c i}$ & $=$ & $\begin{array}{l}\text { swirling-strength, imaginary part of the complex conjugate eigenvalue of } \\
\nabla \mathbf{u} \text {, vortex-identification criterion }\end{array}$ \\
\hline$\lambda_{2}$ & $=$ & $\begin{array}{l}\text { the second largest eigenvalue of } \mathbf{S}^{2}+\boldsymbol{\Omega}^{2} \text {, vortex-identification criterion, cf } \\
\text { Appendix A }\end{array}$ \\
\hline$\sigma(0,1)$ & $=$ & sphere with center at origin and unit radius \\
\hline$\omega$ & $=$ & vorticity vector, $\boldsymbol{\omega}=\nabla \times \mathbf{u}$ \\
\hline$\omega$ & $=$ & vorticity tensor component in $2 \mathrm{D}$ \\
\hline $\boldsymbol{\omega}_{\mathrm{RAVG}}$ & $=$ & $\begin{array}{l}\text { average-corotation vector defined by (12) and (15), and approximated by } \\
\text { (16) }\end{array}$ \\
\hline $\boldsymbol{\omega}_{\mathrm{RES}}, \omega_{\mathrm{RES}}$ & $=$ & $\begin{array}{l}\text { residual-vorticity vector defined by (11), residual-vorticity tensor component } \\
\text { in } 2 \mathrm{D} \text { defined by (10) }\end{array}$ \\
\hline$\omega_{\mathrm{SH}}$ & $=$ & shear vorticity defined (implicitly) by (10) \\
\hline $\boldsymbol{\omega}_{\mathrm{SAVG}}$ & $=$ & average shear vorticity defined by (18) \\
\hline$\Omega$ & $=$ & vorticity tensor, antisymmetric part of $\nabla \mathbf{u}$ \\
\hline$\Omega_{\text {AVERAGE }}$ & $=$ & average angular velocity of line segments, according to Fig. 2 \\
\hline$\Omega_{\mathrm{LOW}}, \Omega_{\mathrm{HIGH}}$ & $=$ & extremal values of angular velocities of line segments, according to Fig. 2 \\
\hline$\Delta \Omega$ & $=$ & $\begin{array}{l}\text { difference of angular velocities of two instantaneously mutually orthogonal } \\
\text { line segments, according to Fig. } 2\end{array}$ \\
\hline
\end{tabular}

Subscripts and superscripts

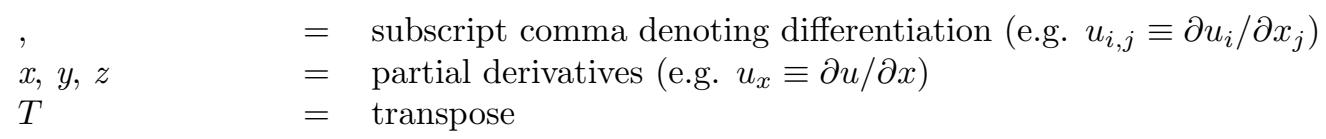

Other symbols and special functions 
$\|\ldots\| \quad=$ absolute tensor value defined by Frobenius norm, $\|\mathbf{G}\|=\left[\operatorname{tr}\left(\mathbf{G G}^{T}\right)\right]^{1 / 2}$

$\operatorname{sgn} \ldots \quad=\quad$ function returning \pm 1 according to the sign of the argument

\section{Introduction}

Various vortex-identification methods [1-22] have been proposed during the last three decades. The search for efficient 3D vortex-identification schemes has become particularly important for the analysis of transitional and turbulent flows, especially in the analysis of LES and DNS data sets. There is an extensive amount of literature on vortex-identification techniques and their comparison, see Appendix A for a summary. Essentially, most vortex-identification schemes can be classified either as a region-type method or a line-type method. Without going into details, the objective of the line-type methods is to identify the vortex-core lines instead of general spatial regions. The region-type and line-type methods may be effectively combined as already shown in previous flow visualization studies [23-25]. A brief explanation of well-established methods related to the present paper is also included in the Appendix A.

Although many of these methods are successful in vortex identification and provide valuable insight into various vortical flows, none of them has become universally applicable to all possible flow situations due to specific limitations. For example, the widely used $\lambda_{2}$-method is valid for incompressible flows only $[6,8]$, and it may cut a connected vortex into broken segments at locations with strong axial stretching [26]. In addition, even for 2D flow cases, $\lambda_{2}$-criterion becomes a shear-biased quantity at regions of high shear when describing inner vortex structure [15]. However, it still remains much more powerful than the conventional vorticity which cannot distinguish between pure shearing motions and the actual swirling motion of a vortex.

An advanced kinematic approach to characterization of local rigid-body like rotational motion is presented below. It is based on (i) the notion of local corotation of material line segments at a point as defined on a two-dimensional plane and (ii) the averaging process applied to 'all planar cross-sections' going through the given point. This procedure gives an easy-to-interpret local (i.e. near a point) vortex intensity as a vector which can be employed in 3D vortex identification.

The basic idea in the design of the proposed approach is to explicitly detect the effect of shear on vorticity and to remove it. To this end, first the notion of local corotation of material line segments is introduced for an arbitrary planar cross-section going through the given point in a 3D flow. Local corotation of line segments near a point is closely related to the interpretation of the so-called residual vorticity in $2 \mathrm{D}$ [15] where different components of vorticity characterize different components of local motion: shear vorticity is associated with shearing motion, and residual vorticity - obtained after the elimination of shearing motion - is associated with rigid-body rotation. Although such elimination is a favorable feature of vortex identification in general, and the widely used methods achieve this goal rather satisfactorily, it is not an explicit requirement of their design and derivation.

Some positive aspects of the direct elimination of local shearing motion has been recently discussed in [27], where the swirling strength criterion $\left(\lambda_{c i}\right)$ [9] is compared to the residual vorticity [15]. In [27], the authors analyzed cross-sections of vortices in turbulent flows, for which their method based on the residual vorticity performed significantly better. The two-dimensional residual vorticity was also found to be a useful tool for shear-layer analysis in [28].

While the residual vorticity is clearly defined by a simple formula in two dimensions, a straightforward and simply computable extension to three-dimensional flows is not readily available. The definition of three-dimensional residual vorticity presented within the triple-decomposition method in [15] uses the basic reference frame $(B R F)$, a special local coordinate system in which the effect of shear is most significant. However, the search for BRF presents an optimization problem for each point in the domain, which makes the triple-decomposition method computationally expensive. Although this issue can be resolved for example by means of parallel computing (e.g. we describe the application of Graphics Processing Unit for this task in [29]), it presents an obstacle for a straightforward practical applicability of the method.

Another approach to using the two-dimensional residual vorticity to analyzing three-dimensional flows was recently presented in [30], where a maximum of residual vorticity was sought over all planes passing through the considered point. While reducing the computational complexity of the BRF search by an order of magnitude, this quantity turned out not to be suited well for vortex identification and provided a reasonable agreement with the established methods only for regions with large intensity of vortical motion. The main reason for the limited applicability of the so called maximum-corotation method is the unstable behavior of the maxima, which can oscillate from point to point and can lead to uneven surfaces of identified structures. 
Moreover, the method is quite sensitive to numerical sampling of planes and for some specific velocity gradient data, it may even suffer from ambiguity regarding the orientation of the maximum-corotation plane.

In this contribution, we present a third, by our opinion the most promising extension of the twodimensional residual vorticity to three dimensions. Instead of searching for maxima over all planes, we use an averaging process, in which each plane is of equal significance and plays its role independently of other planes'. This allows defining the new quantity by integration over a unit sphere, which is in an implementation evaluated by existing efficient quadrature rules, and thus presents almost no computational overhead. The resulting new kinematic quantity, the average corotation of material line segments near a point, provides a vector field suitable for $3 \mathrm{D}$ flow analysis. This shear-free rotational quantity provides an alternative to the shear-biased vorticity vector $\boldsymbol{\omega}=\nabla \times \mathbf{u}$ by correcting it by the effect of shear. At points where no shear is present, the new quantity just reproduces the vorticity field.

The magnitude of the resulting vector of average corotation is used for visualization of vortices in this contribution. It is shown, that the new quantity provides an easy kinematic interpretation and useful qualitative features, which are discussed in connection with widely used local methods $\left(Q, \Delta, \lambda_{2}\right.$, and $\left.\lambda_{c i}\right)$. The proposed average-corotation method remains applicable to compressible and variable-density flows.

Four different flow situations, a hairpin vortex of boundary-layer transition, the reconnection process of two Burgers vortices, a flow around an inclined flat plate, and a flow around a revolving insect wing are analyzed in terms of the average corotation. DNS data sets for the first two flow situations have been provided by IAG, University of Stuttgart. The identified structures are generally in a very good agreement with the existing methods. However, the average corotation seems to better distinguish between local shearing motion and local rigid body rotation (due to swirling motion) in regions of dominant shear, such as 'vortex sheets'.

\section{Local Corotation of Material Line Segments in a Plane}

The first step is the pointwise analysis of the flow on an arbitrary planar cross-section passing through an examined point in a three-dimensional velocity field. Restriction to the plane allows the use of the twodimensional analysis developed in [15], which is recalled in this section. The averaging process for obtaining the final three-dimensional quantity is presented in the next section.

The relative motion near a point where all three velocity components are zero (i.e. near a so-called critical point) can be described by the leading linear terms of a Taylor series expansion of the velocity field in terms of space coordinates (see Chong et al. [4]). The coefficients of the leading linear terms are just elements of the velocity-gradient tensor $\nabla \mathbf{u}$.

Let us select a plane going through a point $\mathbf{x}_{0}$ with the normal vector $\mathbf{n}$. We can introduce a new coordinate system $\left(x^{\prime}, y^{\prime}, z^{\prime}\right)$ with the $z^{\prime}$-axis aligned with $\mathbf{n}$, and axes $x^{\prime}$ and $y^{\prime}$ arbitrarily oriented in the plane. Denoting $\mathbf{Q}$ the orthogonal transformation matrix between the two frames, i.e. $\mathbf{x}^{\prime}=\mathbf{Q x}$, the velocity gradient transformed to the new coordinate system is given as

$$
\widetilde{\nabla \mathbf{u}}=\mathbf{Q}(\nabla \mathbf{u}) \mathbf{Q}^{T}
$$

To analyze the flow behavior on the two-dimensional cross-section aligned with the $x^{\prime} y^{\prime}$-plane, it is sufficient to look at the leading $2 \times 2$ submatrix of $\widetilde{\nabla \mathbf{u}}$.

In general, the $2 \times 2$ submatrix

$$
\left(\begin{array}{ll}
u_{x} & u_{y} \\
v_{x} & v_{y}
\end{array}\right)
$$

is associated with a standard two-dimensional velocity gradient, where now $x, y$ and $u, v$ refer to $2 \mathrm{D}$ coordinates and velocity components, respectively, and the subscripts stand for partial derivatives. The infinitesimal velocity contributions due to the linearization near a reference point are in Fig. 1 explicitly stated by the arrows associated with the rotational motion in 2D near a point $P$. Apparently, these velocity contributions are 'attached' to the instantaneous infinitesimal line segments passing through the reference point. Figure 2 focuses on the rotation of these line segments which, at a given instant of time, rotate at generally different angular speeds (positive counterclockwise orientation). These angular velocities can be inferred from the leading linear terms, explicitly shown in Fig. 1, by the elementary relationship between the angular and the tangential velocity. Vorticity, expressing an average angular velocity of fluid elements, is represented in

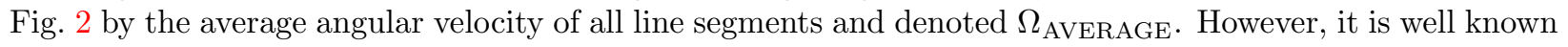




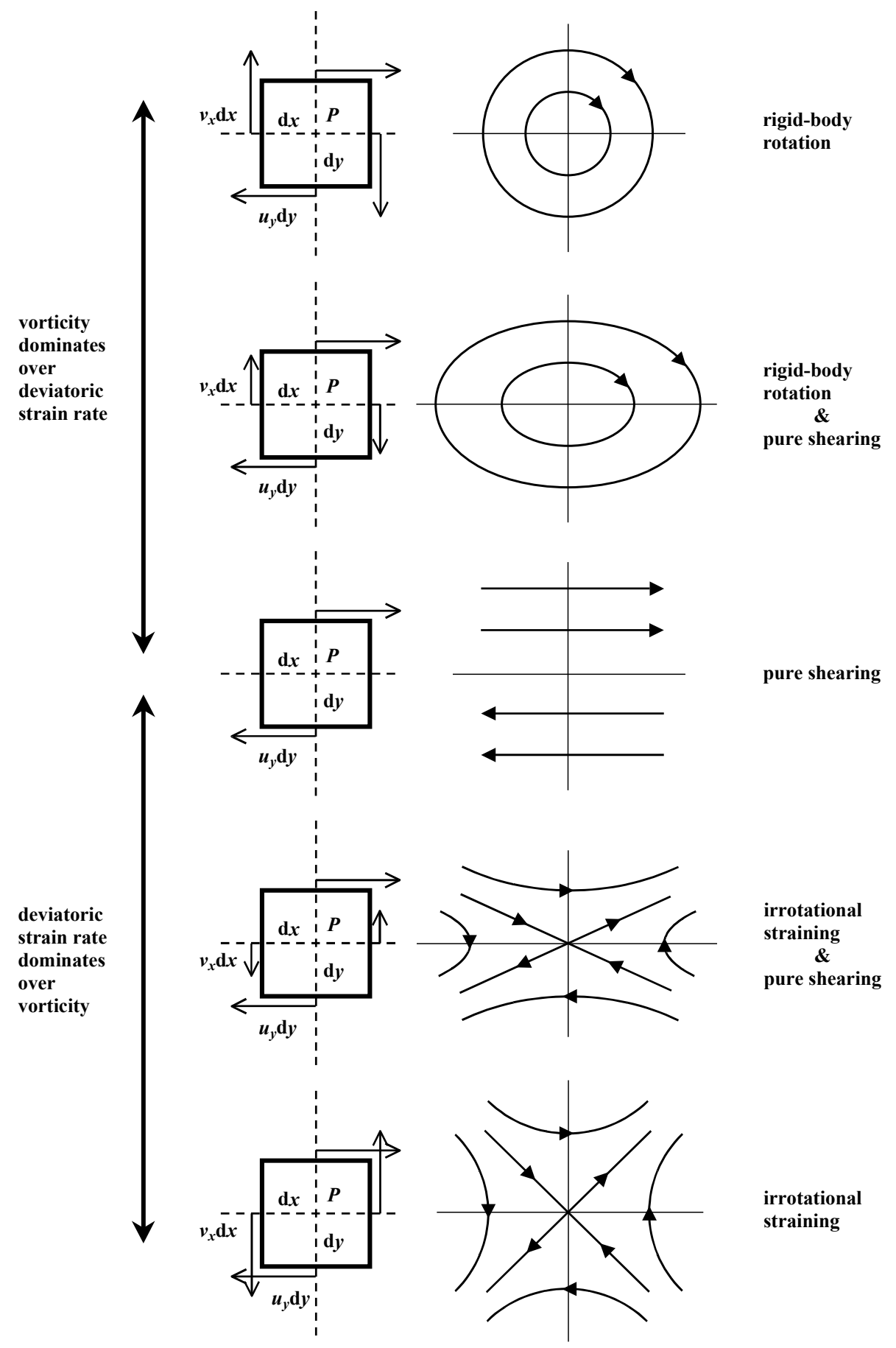

Figure 1: Local flow patterns of deviatoric motion near a point (according to [15]): Dashed line segments are associated with the instantaneous extremal angular velocities found over all line segments going through a point $P$. 
instantaneously mutually orthogonal line segments fulfilling: $|\Delta \boldsymbol{\Omega}|=$ MAXIMUM $=\left|\boldsymbol{\Omega}_{\text {HIGH }}-\boldsymbol{\Omega}_{\text {LOW }}\right|$ formally assuming $\left|\Omega_{\mathrm{HIGH}}\right| \geq\left|\boldsymbol{\Omega}_{\mathrm{LOW}}\right|$
$\left|\Omega_{\text {HIGH }}\right|-\left|\Omega_{\text {LOW }}\right|>0$ for both cases

(i.e. corotation and contrarotation)

$\Rightarrow$ shearing motion

vorticity dominates over deviatoric strain rate

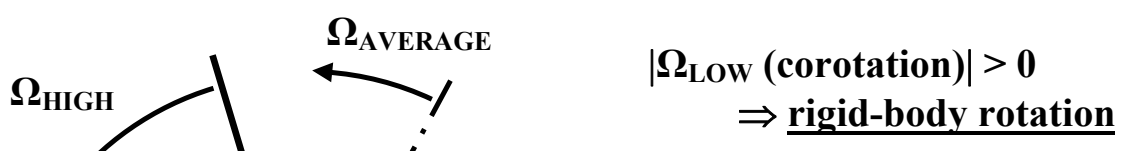

deviatoric strain rate dominates over vorticity

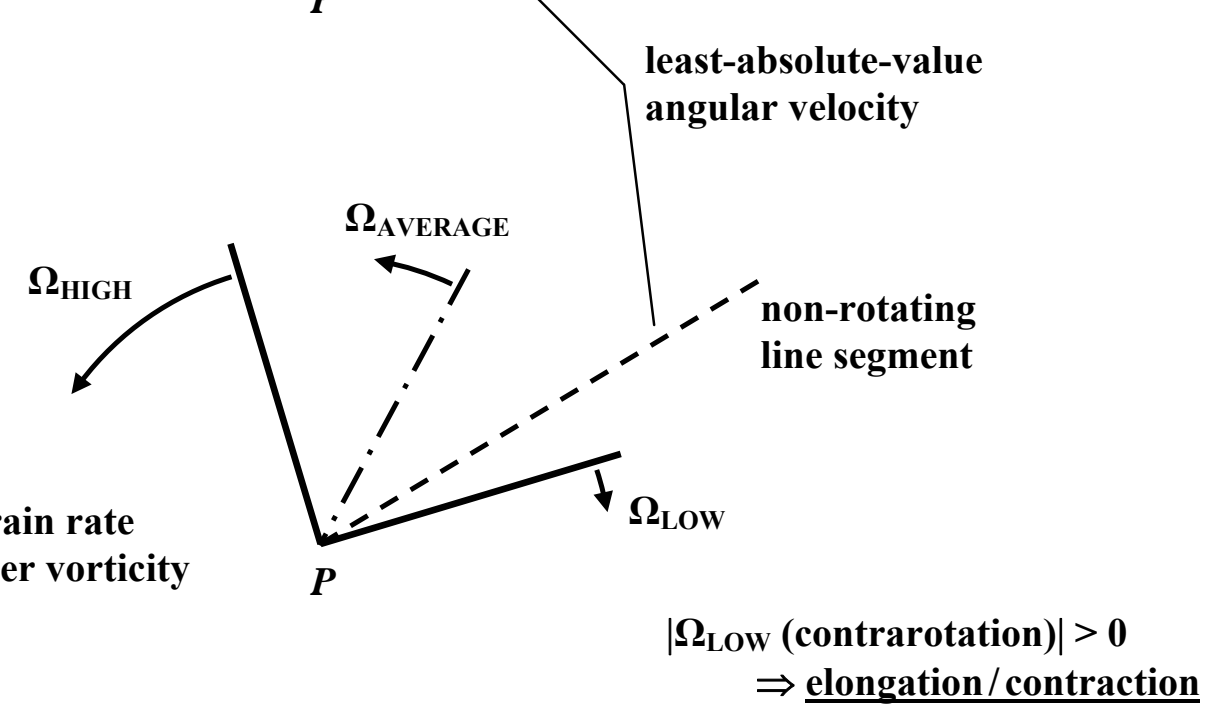

Figure 2: Corotation of line segments: The residual vorticity is shown in terms of the least-absolute-value angular velocity. 
that this quantity cannot distinguish between shearing motions and the actual swirling motion of a vortex and misrepresents vortex geometry.

The planar residual vorticity obtained after the removal of shearing motion is the least-absolute-value angular velocity of all the infinitesimal line segments passing through the given point, see Fig. 2. This quantity represents nothing but a measure of the instantaneous local rigid-body rotation found on the selected planar cross-section (see also Appendix B).

As can be inferred from Fig. 2, the planar residual vorticity - a measure of the local rigid-body rotation-is nonzero only if all of the line segments corotate. Local corotation is then quantified just in terms of the planar residual vorticity. The quantity $\Omega_{\text {AVERAGE }}$ in Fig. 2 represents the conventional vorticity $\omega$, while $\Omega_{\mathrm{HIGH}}$ and $\Omega_{\text {LOW }}$ stand for the extremal values of angular velocity (including sign!) found over all line segments going through point $P$. The extremal angular velocities $\Omega_{\mathrm{HIGH}}$ and $\Omega_{\mathrm{LOW}}$ are defined by the requirements framed in Fig. 2. The local flow patterns shown in Fig. 1 hold for a planar deviatoric motion which is sufficient for the corotation considerations on an arbitrary planar cross-section in 3D flow. To quantify the local corotation, the planar residual vorticity $\omega_{\mathrm{RES}}$ is employed. As mentioned above, this is interpreted in terms of the least-absolute-value angular velocity of all infinitesimal line segments (subsets of the plane) near a point, passing through the given point. The relevant quantities necessary for the determination of $\omega_{\mathrm{RES}}$ are the vorticity $\omega$ and the planar deviatoric (i.e. traceless) principal strain-rate magnitude $\left|s_{D}\right|$.

For a general non-deviatoric $2 \times 2$ submatrix (2), the planar deviatoric strain-rate tensor $\left(s_{i j}\right)_{D}$ is obtained from the symmetric part by subtracting half of the trace from diagonal entries,

$$
\left(s_{i j}\right)_{D}=\left(\begin{array}{cc}
u_{x}-\left(u_{x}+v_{y}\right) / 2 & \left(u_{y}+v_{x}\right) / 2 \\
\left(u_{y}+v_{x}\right) / 2 & v_{y}-\left(u_{x}+v_{y}\right) / 2
\end{array}\right) .
$$

In the system of principal axes, tensor $\left(s_{i j}\right)_{D}$ corresponds to the diagonal matrix

$$
\left(\begin{array}{cc}
s_{D} & 0 \\
0 & -s_{D}
\end{array}\right)=\left(\begin{array}{cc} 
\pm\left(\sqrt{\left(u_{x}-v_{y}\right)^{2}+\left(u_{y}+v_{x}\right)^{2}}\right) / 2 & 0 \\
0 & \mp\left(\sqrt{\left(u_{x}-v_{y}\right)^{2}+\left(u_{y}+v_{x}\right)^{2}}\right) / 2
\end{array}\right)
$$

and so

$$
\left|s_{D}\right|=\left(\sqrt{\left(u_{x}-v_{y}\right)^{2}+\left(u_{y}+v_{x}\right)^{2}}\right) / 2 .
$$

Vorticity $\omega$ and planar deviatoric principal strain-rate magnitude $\left|s_{D}\right|$ can be unambiguously expressed in terms of angular velocities near the point depicted in Fig. 2. First,

$$
\omega=\left(v_{x}-u_{y}\right) / 2=\left(\Omega_{\mathrm{HIGH}}+\Omega_{\mathrm{LOW}}\right) / 2=\Omega_{\mathrm{AVERAGE}} .
$$

For the corotation case, where vorticity dominates over deviatoric strain rate $\left(|\omega|>\left|s_{D}\right|\right)$,

$$
\left|s_{D}\right|=\left(\left|\Omega_{\mathrm{HIGH}}\right|-\left|\Omega_{\mathrm{LOW}}\right|\right) / 2 .
$$

Alternatively, for the contrarotation case, where deviatoric strain rate dominates over vorticity $\left(|\omega|<\left|s_{D}\right|\right)$,

$$
\left|s_{D}\right|=\left(\left|\Omega_{\mathrm{HIGH}}\right|+\left|\Omega_{\mathrm{LOW}}\right|\right) / 2 \text {. }
$$

Finally, for pure shearing $\left(|\omega|=\left|s_{D}\right|, \Omega_{\mathrm{LOW}}=0\right)$,

$$
\left|s_{D}\right|=\left|\Omega_{\mathrm{HIGH}}\right| / 2 \text {. }
$$

The key quantity of the corotation approach, the residual vorticity $\omega_{\mathrm{RES}}$, reads ([15], for details see Appendix B)

$$
\omega_{\mathrm{RES}}= \begin{cases}\omega-\omega_{\mathrm{SH}}=(\operatorname{sgn} \omega)\left(|\omega|-\left|s_{D}\right|\right)=\Omega_{\mathrm{LOW}} & \text { for }|\omega| \geq\left|s_{D}\right|, \\ 0 & \text { for }|\omega| \leq\left|s_{D}\right|,\end{cases}
$$

where $\omega_{\text {SH }}$ stands for the vorticity due to shear.

As follows from (3)-(6), uniform dilatation (or contraction) appearing through the $2 \times 2$ submatrix of the velocity gradient tensor and given on the examined plane by $\left(u_{x}+v_{y}\right) / 2$, i.e. by half of the trace of the submatrix, affects neither the shape nor the rotational characteristics $\left|s_{D}\right|$ and $\omega$. Consequently, it does 
not affect our corotation measure, the residual vorticity $\omega_{\mathrm{RES}}$. That is why a planar deviatoric motion is sufficient for the present procedure.

Let us now turn back to the general 3D setting and the original coordinate system. The planar local corotation has vector character, with direction of the normal of the examined plane $\mathbf{n}$ and magnitude $2 \omega_{\mathrm{RES}}$, where $\omega_{\text {RES }}$ is given by (10). The factor 2 is related to using $\frac{1}{2}$ in the expression for the two-dimensional vorticity tensor component in (6). As a function of both the selected point $\mathbf{x}_{0}$ and the orientation of the plane normal $\mathbf{n}$, the local corotation vector is expressed in $3 \mathrm{D}$ as

$$
\boldsymbol{\omega}_{\mathrm{RES}}\left(\mathbf{x}_{0}, \mathbf{n}\right)=2 \omega_{\mathrm{RES}} \mathbf{n}
$$

\section{Determination of Average Corotation}

The determination of average corotation is a pointwise procedure. For a given position vector $\mathbf{x}_{0}$ the averaging procedure is applied over 'all planar cross-sections'. A convenient way to formalize and compute the average corotation is based on surface integration. Keeping in mind the key role of a normal $\mathbf{n}$ in the present averaging process, the set of all planes going through the given point can be redefined as the set of all tangential planes of a unit sphere $\sigma(0,1)$. Consequently, the average-corotation vector at $\mathbf{x}_{0}$ is defined as

$$
\boldsymbol{\omega}_{\mathrm{RAVG}}\left(\mathbf{x}_{0}\right)=\alpha \frac{\iint_{\sigma(0,1)} \boldsymbol{\omega}_{\mathrm{RES}}\left(\mathbf{x}_{0}, \mathbf{n}\right) \mathrm{d} \sigma}{\iint_{\sigma(0,1)} \mathrm{d} \sigma}=\frac{\alpha}{4 \pi} \iint_{\sigma(0,1)} \boldsymbol{\omega}_{\mathrm{RES}}\left(\mathbf{x}_{0}, \mathbf{n}\right) \mathrm{d} \sigma,
$$

where $\alpha \in \mathbb{R}$ is a scaling factor. A natural choice $\alpha=3$ is derived in Appendix C.

A considerable amount of research has been devoted to efficient numerical evaluation of surface integrals over the unit sphere such as (12), see e.g. [31], [32], or an overview paper [33]. While these methods can lead to very fast evaluation of average corotation, they are somewhat complicated and technical. For the purpose of the present paper, the basic rectangle integration scheme is employed further. However, suitability of some more advanced schemes is briefly discussed at the end of Section 6.

It should be noted that (12) is not a surface integral of a vector-valued function as is well known e.g. from continuum mechanics. Instead, (12) presents three independent surface integrals of scalar functions, one for each component of the average corotation. Also note, that no temporal or spatial averaging is involved in the procedure in (12).

Using the spherical coordinates $r, \varphi, \vartheta$, and

$$
x_{1}=r \sin \vartheta \cos \varphi, x_{2}=r \sin \vartheta \sin \varphi, x_{3}=r \cos \vartheta
$$

the surface element on a unit sphere with $r=1$ can be expressed as

$$
\mathrm{d} \sigma=\sin \vartheta \mathrm{d} \vartheta \mathrm{d} \varphi
$$

For $\varphi \in[0,2 \pi], \vartheta \in[0, \pi]$, the surface integral in (12) can be transformed using Fubini's theorem into a double integral

$$
\boldsymbol{\omega}_{\mathrm{RAVG}}\left(\mathbf{x}_{0}\right)=\frac{\alpha}{4 \pi} \int_{0}^{2 \pi} \int_{0}^{\pi} \boldsymbol{\omega}_{\mathrm{RES}}\left(\mathbf{x}_{0}, \mathbf{n}(\varphi, \vartheta)\right) \sin \vartheta \mathrm{d} \vartheta \mathrm{d} \varphi .
$$

The integral (15) is evaluated numerically by subdividing the domain $[0,2 \pi] \times[0, \pi]$ into squares with the size $\delta$ so that the number of intervals is $N_{\varphi}=2 \pi / \delta$ and $N_{\vartheta}=\pi / \delta$. For each of these squares, the normal $\mathbf{n} \equiv \mathbf{n}(\varphi, \vartheta)$ and the corresponding $\boldsymbol{\omega}_{\mathrm{RES}}\left(\mathbf{x}_{0}, \mathbf{n}(\varphi, \vartheta)\right)$ are approximated as constant vectors. The final expression of average-corotation vector employed for further calculations reads

$$
\boldsymbol{\omega}_{\mathrm{RAVG}}\left(\mathbf{x}_{0}\right) \approx \frac{\alpha}{4 \pi} \sum_{j=1}^{N_{\varphi}} \sum_{i=1}^{N_{\vartheta}} \boldsymbol{\omega}_{\mathrm{RES}}\left(\mathbf{x}_{0}, \mathbf{n}\left(\varphi_{j}, \vartheta_{i}\right)\right) \sin \vartheta_{i} \delta^{2}
$$


The average-corotation vector $\boldsymbol{\omega}_{\mathrm{RAVG}}$ can be expressed in terms of its magnitude $\left|\boldsymbol{\omega}_{\mathrm{RAVG}}\right|$ and directiondenoted $\mathbf{n}_{\text {SWIRL }}$ since it defines the resulting 'plane of swirling' - as functions of the only independent variable, a position $\mathbf{x}_{0}$ in $3 \mathrm{D}$ flow field, as

$$
\boldsymbol{\omega}_{\mathrm{RAVG}}\left(\mathbf{x}_{0}\right)=\left|\boldsymbol{\omega}_{\mathrm{RAVG}}\left(\mathbf{x}_{0}\right)\right| \mathbf{n}_{\mathrm{SWIRL}}\left(\mathbf{x}_{0}\right) .
$$

Note that the present average-corotation method and the earlier maximum-corotation method [30] (also shown below), which is based on the maximum corotation found over 'all planar cross-sections', are similar in that they both search over 'all planes' given by the normal $\mathbf{n} \equiv \mathbf{n}(\varphi, \vartheta)$ on the domain $[0,2 \pi] \times[0, \pi]$. Consequently, both methods lead to a very similar computational cost. However, a significant advantage of the average-corotation method over the maximum-corotation method consists in the applicability of more efficient integration schemes.

It should be emphasized that the averaging procedure introduced in the present paper represents in fact a normalized additive process by adding information 'planar cross-section by planar cross-section' to account properly for a full 3D kinematic picture near a point. One selected planar information, even though choosing the maximum-corotation plane, degenerates the overall kinematic information and the local flow behavior near a point cannot be taken in a truly 3D representative manner.

\section{Applications of Average Corotation to Vortex Identification}

Four different flow situations are considered: a hairpin vortex of boundary-layer transition, the reconnection process of two Burgers vortices, a flow around an impulsively started flat plate at an angle of attack of $30^{\circ}$, and a flow around a revolving insect wing. These flows are examined in terms of average corotation. For comparison purposes, the popular $\lambda_{2}$-criterion and the maximum-corotation method are evaluated simultaneously with the average-corotation method for the first two applications. The third application is focused on the shearing bias in vortex identification and compares the average-corotation method with $\lambda_{2}$-criterion and $Q$-criterion (for definitions see Appendix A). The fourth application further extends the study of the bias due to shearing.

\section{Hairpin vortex of boundary-layer transition}

Boundary-layer transition belongs to basic flow problems associated with distinct vortical structures. The examined DNS data set deals with numerical simulation of wind-tunnel experiments using controlled disturbance excitation with frequency $=62.5 \mathrm{~Hz}$ at $\mathrm{Re}$ [based on the displacement thickness] $=730$, according to [34]. Figure 3 shows a single representative hairpin vortex formed during this transition process in terms of different criteria. We present the detected vortical structures by plotting isosurfaces at an increasing value of the magnitude of average corotation. This value is called threshold and reported in percents of the maxima over the domain (minima for $\lambda_{2}$ ).

To provide a comparable plot for different methods, the following matching procedure is performed. After choosing a threshold for $\left|\boldsymbol{\omega}_{\mathrm{RAVG}}\right|$, we find corresponding thresholds for the other methods by minimizing the characteristic ratio $V_{N} / V_{O}$ over all possible thresholds. Here, $V_{N}$ corresponds to the volume where methods do not overlap in terms of vortex regions (i.e. one method detects a vortex while the other does not) and $V_{O}$ denotes the overlapping volume where both methods detect a vortex region.

Figure 3 indicates a close resemblance of the average-corotation results with $\lambda_{2}$-method for $\lambda_{2}$-threshold of $2.6 \%$ and higher, while identifying slightly different structure at the lowest threshold. On the other hand, the maximum-corotation scheme is apparently less satisfactory, with the exception of the highest threshold.

\section{Reconnection process of two Burgers vortices}

The second application deals with the subsonic DNS data sets for the reconnection process of two Burgers vortices at $\mathrm{Ma}=0.3$ and $\mathrm{Ma}=0.8$, at $\mathrm{Re}[\equiv$ circulation/kinematic viscosity $]=10000$. The mutual matching of plots in Figs. 4 and 5 is also performed for fixed average-corotation thresholds followed by minimizing of the characteristic ratio $V_{N} / V_{O}$ over all possible thresholds.

As shown in Figs. 4 and 5, the mutual similarity of results for all three methods is very good, especially in the way how the three different methods indicate the vortical substructures of connecting ribs of the 

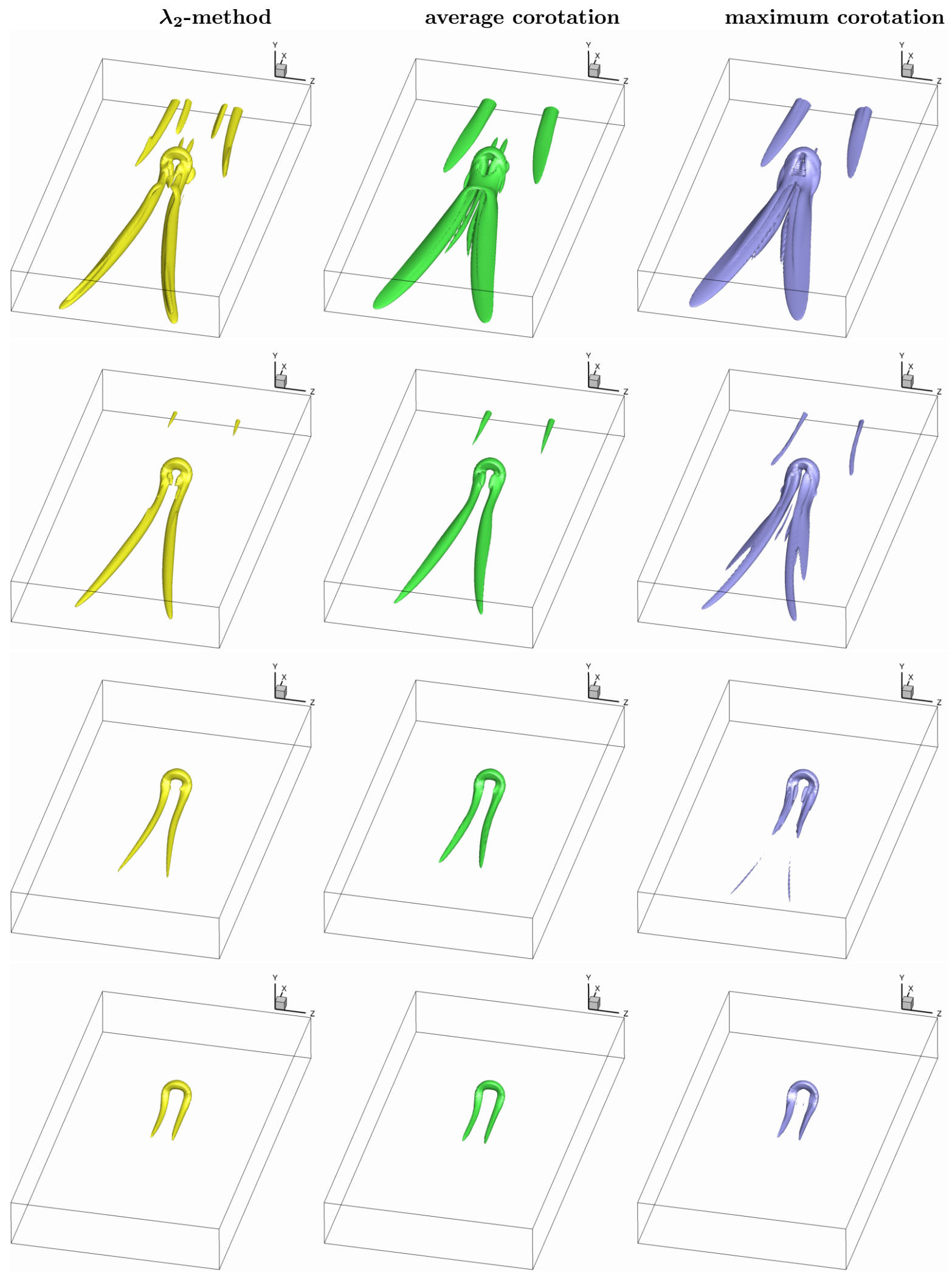

Figure 3: The results for a hairpin vortex at four different relative thresholds for each of the following methods: $\lambda_{2}$-method (left, threshold: $0.6 \%, 2.6 \%, 6.9 \%, 15.2 \%$ ), average-corotation method (center, threshold: $2.7 \%, 10.0 \%, 17.6 \%, 30.6 \%$ ), and maximum-corotation method (right, threshold: $9.0 \%, 16.8 \%, 29.1 \%$, $35.9 \%)$. 
Table 1: The absolute values of threshold for individual methods in Figs. 7 and 8, and the lowest relative non-overlap $V_{N} / V_{O}$ achieved by the matching procedure (in parentheses). The non-overlapping volume $V_{N}$ corresponds to the sum of all regions where only one criterion detects a vortex, while the overlapping volume $V_{O}$ corresponds to the sum of all regions where both criteria detect a vortex. Smaller value of $V_{N} / V_{O}$ corresponds to better agreement.

\begin{tabular}{|c|c|cc|}
\hline Re & $\lambda_{2}$ & $Q$ & $\left|\boldsymbol{\omega}_{\mathrm{RAVG}}\right|$ \\
\hline 300 & -2.0 & $2.01(\mathbf{0 . 0 8})$ & $2.02(\mathbf{0 . 7 2})$ \\
1200 & -2.0 & $2.29(\mathbf{0 . 2 4})$ & $1.73(\mathbf{0 . 3 6})$ \\
\hline
\end{tabular}

reconnection process. However, we are aware, that the applicability of the $\lambda_{2}$-criterion to compressible flows at higher Mach numbers is questionable [8].

\section{Flow around a flat plate at an angle of attack}

The investigated data sets describe the impulsively started incompressible flow around a flat plate (aspect ratio 2) at an angle of attack of $30^{\circ}$ solved numerically for Reynolds numbers $\mathrm{Re}=300$ and $\mathrm{Re}=1200$. The results are shown in Figs. 6-8. This application aims to illustrate how the average-corotation scheme compares with the $\lambda_{2}$-method. Using two different thresholds for $\lambda_{2}$, Figure 6 demonstrates that the average-corotation method is able to capture more universally the vortical structures both in close proximity of the plate and in the wake downstream. In particular, should vortical structures downstream in the wake obtained by a selected value of $\left|\boldsymbol{\omega}_{\mathrm{RAVG}}\right|$ be identified by the $\lambda_{2}$-criterion, a lower threshold of $\lambda_{2}$ is needed, and undesirable shearing zones around the plate edges appear. By taking a higher threshold value to diminish the shearing zones, the relevant downstream vortices disappear completely. This observation holds for both Reynolds numbers under consideration.

In addition, Figure 7 shows a similar situation including the widely used $Q$-criterion. In Fig. 7, we plot the isosurfaces of $\lambda_{2}=-2$, and isosurfaces of the $Q$-criterion and average corotation determined by volume matching procedure described in the previous section, namely minimizing the relative non-overlap $V_{N} / V_{O}$. The values of resulting thresholds and corresponding values of $V_{N} / V_{O}$ are summarized in Table 1 . Note that $V_{N} / V_{O}$ provides a measure of the quality of the match, with lower values corresponding to better agreement.

The results by the $Q$-criterion are very similar to those by $\lambda_{2}$-method, especially for $\mathrm{Re}=300$. Hence, a similar shearing bias in vortex identification appears. To capture the vortical structures in the wake downstream, both the $Q$-criterion and the $\lambda_{2}$-criterion cannot avoid the bias in terms of showing shearing zones around the plate edges as vortex zones. These results are in a good agreement with [35], where the same flow situation was analyzed by means of the earlier (and much more computationally expensive) triple-decomposition method [15].

To indicate the role of shearing motion in the proximity of the plate, we introduce the vector of average shear vorticity $\boldsymbol{\omega}_{\mathrm{SAVG}}$ simply as the difference between the vorticity vector $\boldsymbol{\omega}$ and the average corotation $\boldsymbol{\omega}_{\mathrm{RAVG}}$,

$$
\boldsymbol{\omega}_{\mathrm{SAVG}}=\boldsymbol{\omega}-\boldsymbol{\omega}_{\mathrm{RAVG}}
$$

The same quantity could be also obtained by the averaging procedure analogous to (12) applied to $\boldsymbol{\omega}_{\mathrm{SH}}=$ $2 \omega_{\mathrm{SH}} \mathbf{n}$ in place of $\boldsymbol{\omega}_{\mathrm{RES}}$, where $\omega_{\mathrm{SH}}$ is the planar shear vorticity defined implicitly in (10). Selected isosurfaces of the magnitude of $\boldsymbol{\omega}_{\mathrm{SAVG}}$ are depicted along the results by $\lambda_{2}$-criterion and $\boldsymbol{\omega}_{\mathrm{RAVG}}$ in Fig. 8 . The thresholds for $\lambda_{2}$ and $\boldsymbol{\omega}_{\mathrm{RAVG}}$ are the same as in Fig. 7 . It can be seen, that behind the plate edges, the vortex structures identified by the $\lambda_{2}$-criterion resemble the structure of the shearing zones, unlike the results by $\boldsymbol{\omega}_{\mathrm{RAVG}}$. The fact that $\left|\boldsymbol{\omega}_{\mathrm{SAVG}}\right|$ is significant also inside the vortex regions farther downstream can be attributed to shearing of inner ('concentric cylindrical') vortex layers.

\section{Flow around a revolving insect wing}

The final analyzed data set comprises a model of a wing of a fruit fly (Drosophila), which revolves in a propeller-like motion. The angle of attack is fixed to $40^{\circ}$ and the Reynolds number [based on the velocity of the tip of the wing] Re=500. The vortex structure behind the wing at the end of the first revolution is examined. The whole structure is dominated by the spiralling tip vortex, enclosed by the starting leadingedge and trailing-edge vortices, and by a weaker vortex near the root of the wing. 

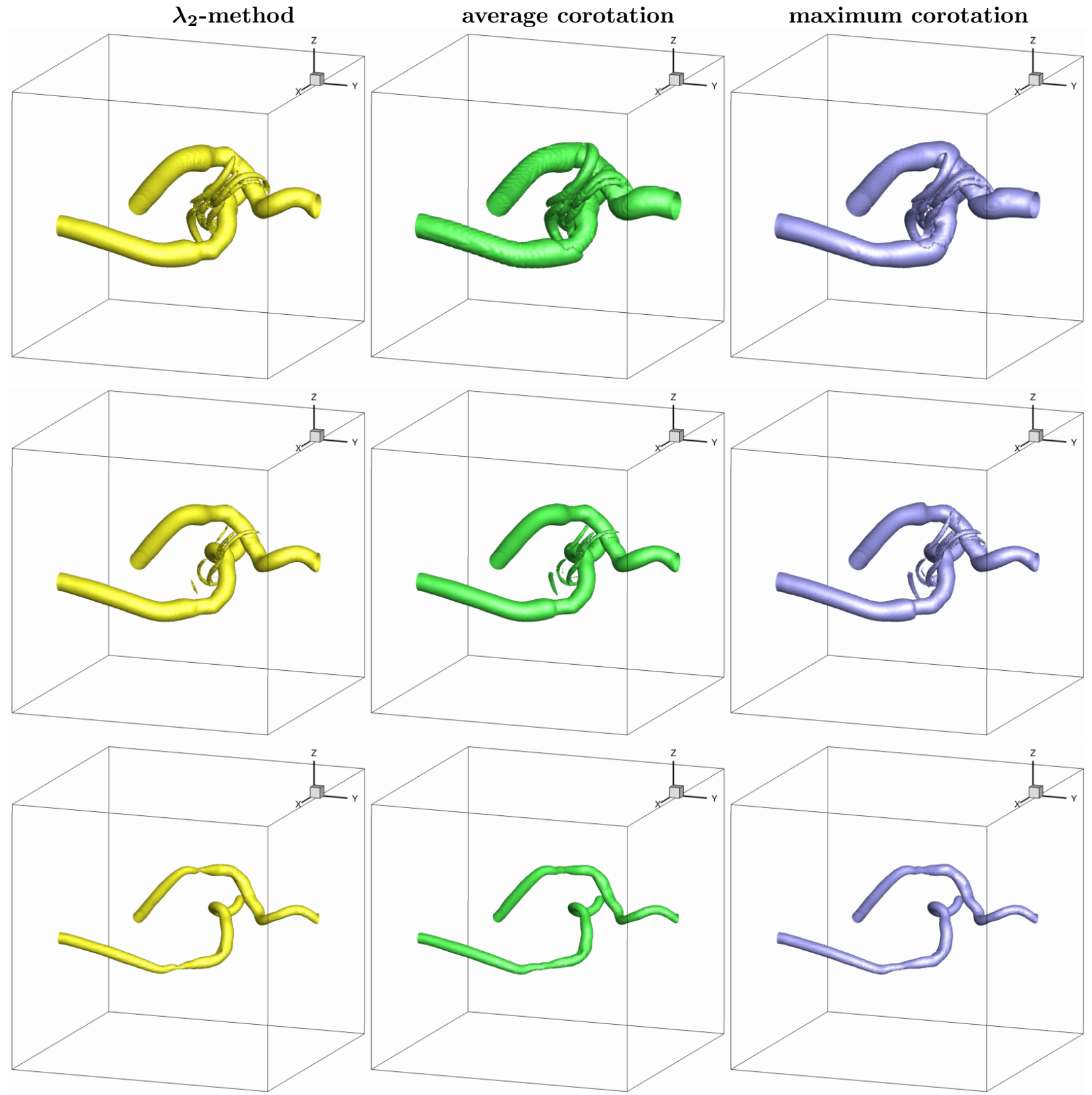

Figure 4: The results for a reconnection of Burgers vortices, $\mathrm{Ma}=0.3$, at three different relative thresholds for each of the following methods: $\lambda_{2}$-method (left, threshold: $0.1 \%, 4.6 \%, 23.1 \%$ ), average-corotation method (center, threshold: $0.8 \%, 13.9 \%, 47.5 \%$ ), and maximum-corotation method (right, threshold: $6.1 \%, 17.0 \%$, $47.7 \%)$. 

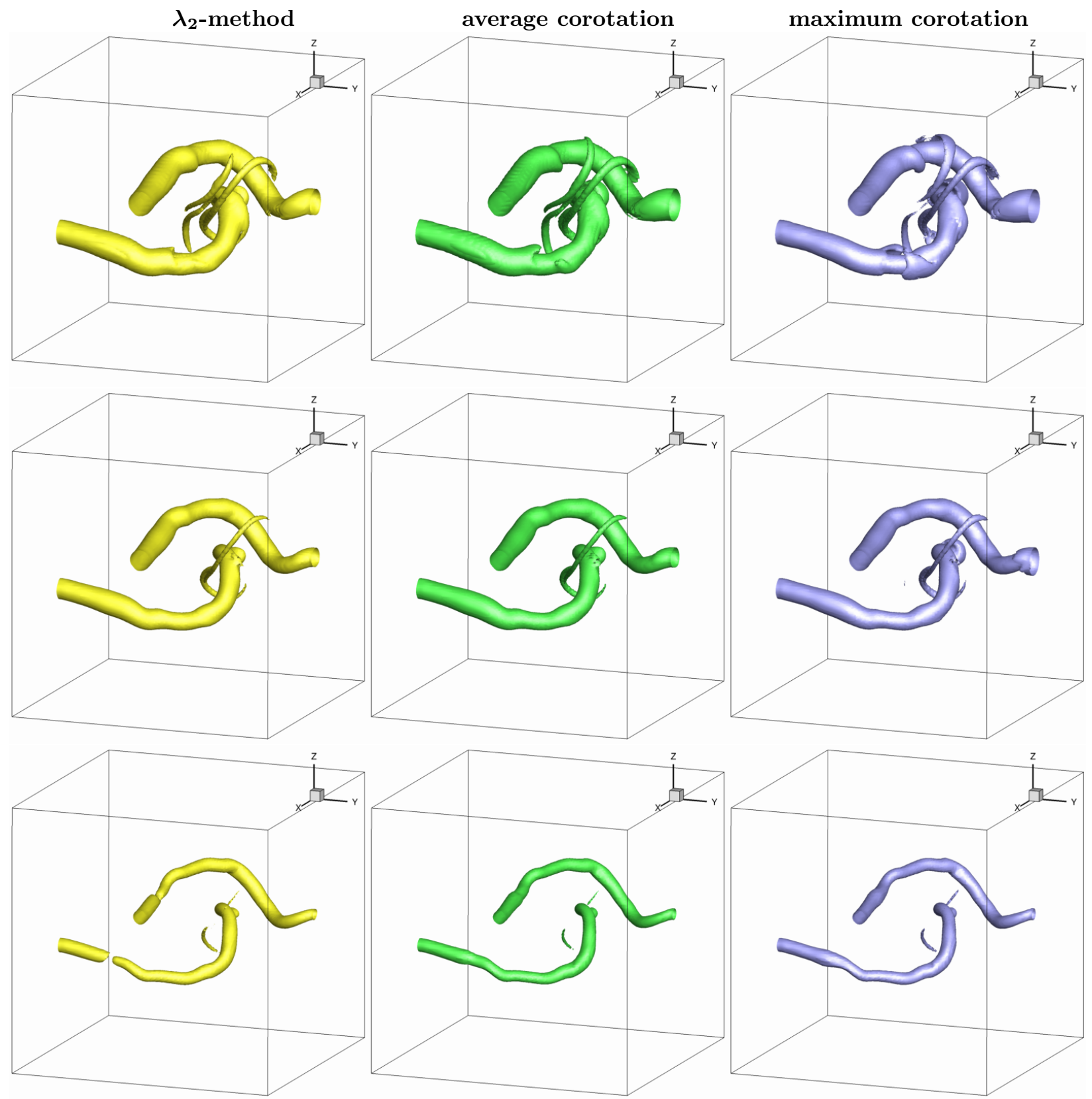

Figure 5: The results for a reconnection of Burgers vortices, $\mathrm{Ma}=0.8$, at three different relative thresholds for each of the following methods: $\lambda_{2}$-criterion (left, threshold: $0.1 \%, 6.1 \%, 24.6 \%$ ), average-corotation method (center, threshold: $2.9 \%, 15.4 \%, 46.3 \%$ ), and maximum-corotation method (right, threshold: 10.1\%, 19.4\%, $46.7 \%)$. 

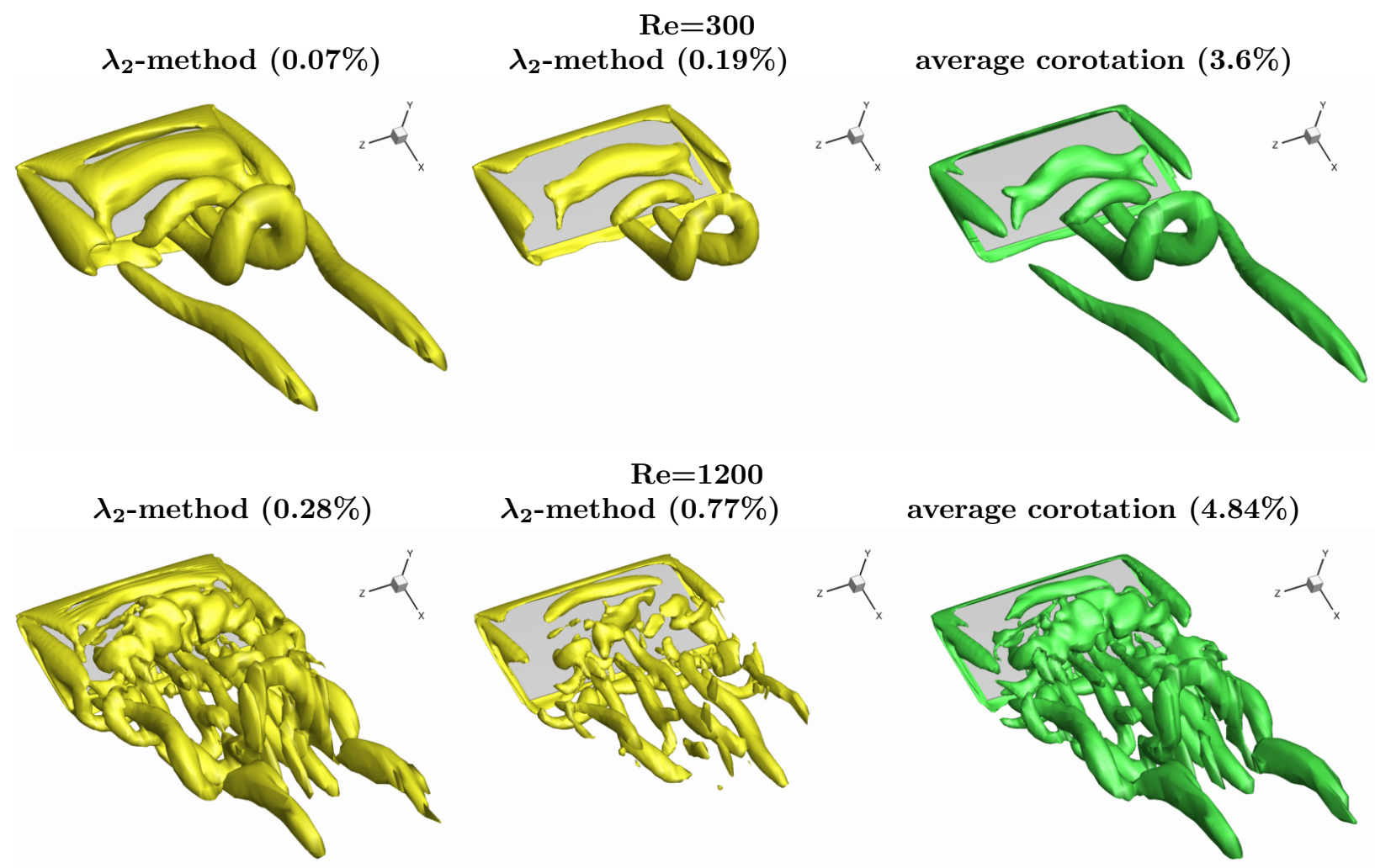

Figure 6: Results for the flow around a flat plate at an angle of attack of $30^{\circ}$ revealing a shearing bias of the $\lambda_{2}$-criterion near the plate edges. The corresponding thresholds are shown in parentheses. 

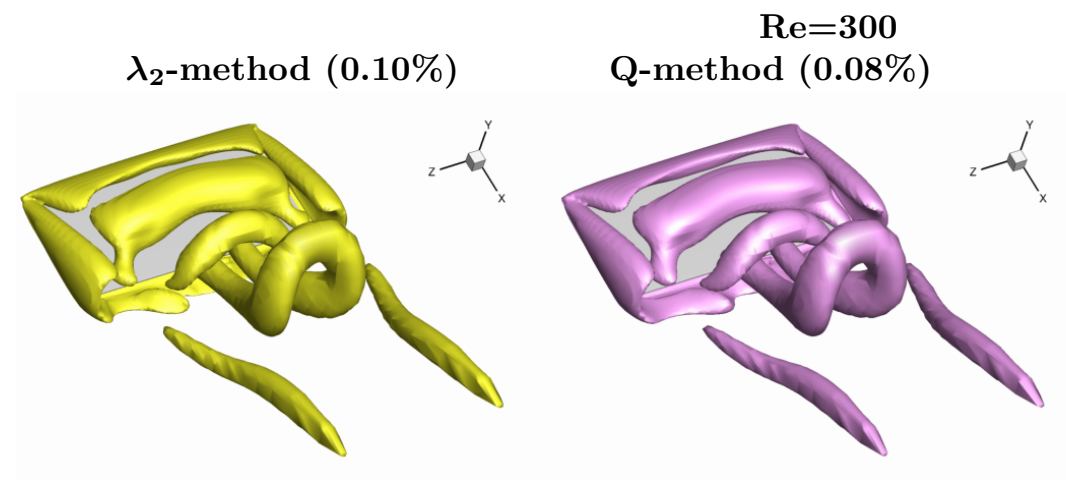

average corotation $(4.0 \%)$
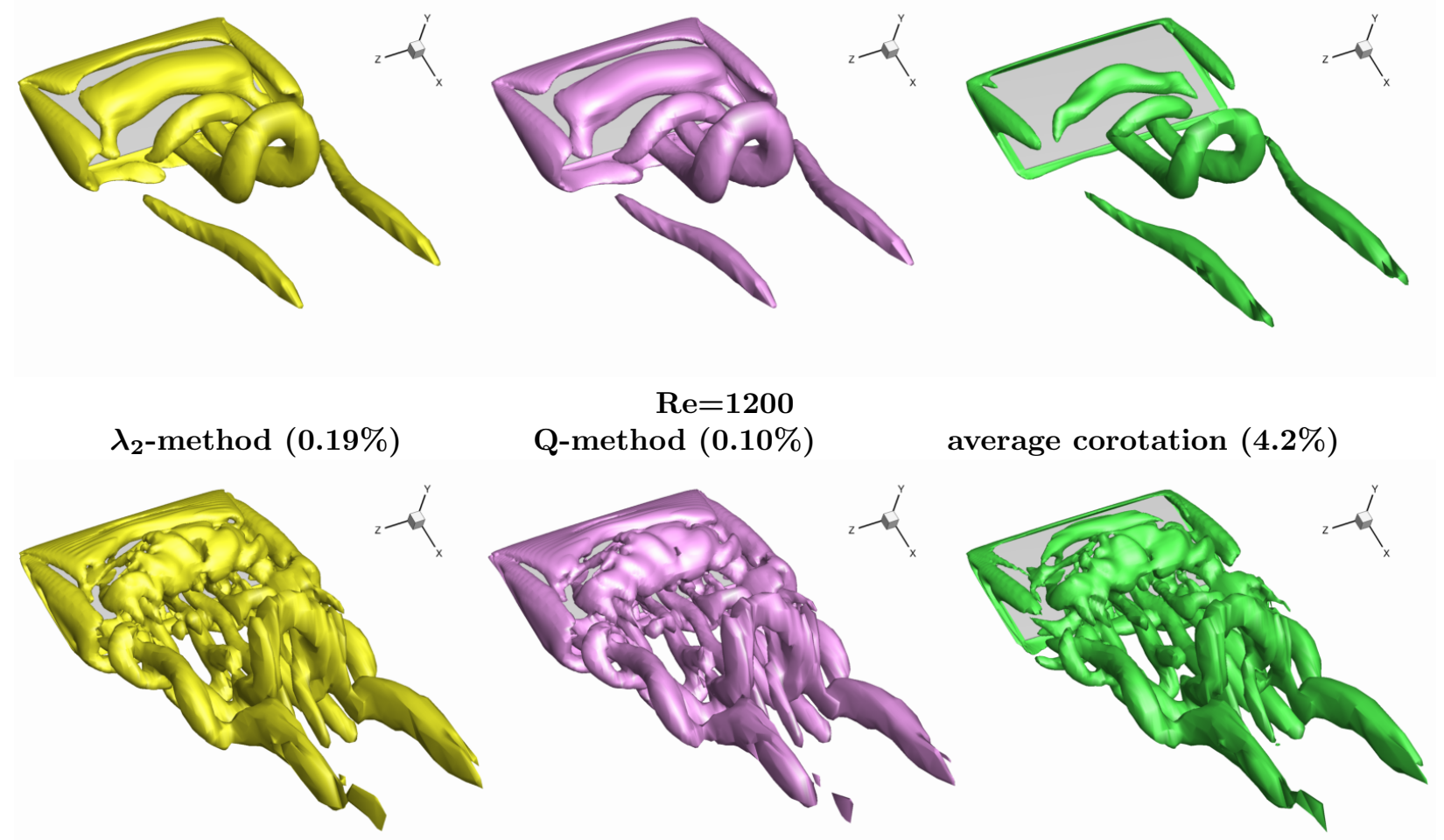

Figure 7: Results for the flow around a flat plate at an angle of attack of $30^{\circ}$ revealing a shearing bias of the $Q$-criterion near the plate edges. The corresponding thresholds are shown in parentheses and in Table 1. 


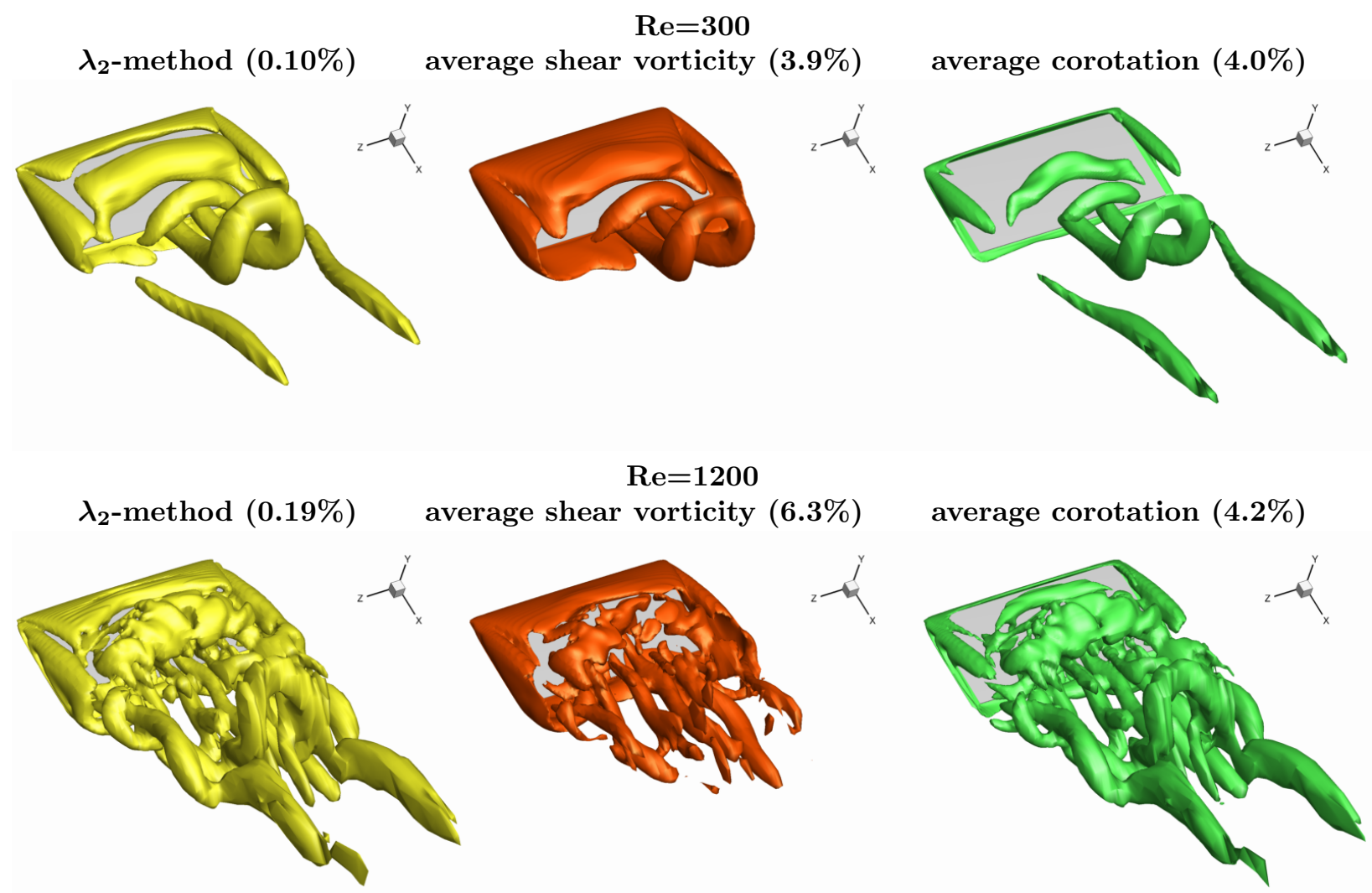

Figure 8: Magnitude of average shear vorticity $\left|\boldsymbol{\omega}_{\mathrm{SAVG}}\right|$ for the flow around a flat plate at an angle of attack of $30^{\circ}$ studying the effect of shearing motion on $\lambda_{2}$-criterion in the vicinity of the plate edges. The corresponding thresholds are shown in parentheses and in Table 1. 
Table 2: The absolute values of threshold for individual methods in Figs. 9 and 10, and the lowest relative non-overlap $V_{N} / V_{O}$ achieved by the matching procedure (in parentheses).

\begin{tabular}{|c|cc|c|cc|}
\hline \multicolumn{3}{|c|}{$\begin{array}{c}\text { Figure } 9 \\
\text { match to } \lambda_{2}\end{array}$} & \multicolumn{3}{c|}{$\begin{array}{c}\text { Figure } 10 \\
\text { match to }\left|\boldsymbol{\omega}_{\mathrm{RAVG}}\right|\end{array}$} \\
\hline$\lambda_{2}$ & $\left|\boldsymbol{\omega}_{\mathrm{SAVG}}\right|$ & $\left|\boldsymbol{\omega}_{\mathrm{RAVG}}\right|$ & $\left|\boldsymbol{\omega}_{\mathrm{RAVG}}\right|$ & $\left|\boldsymbol{\omega}_{\mathrm{SAVG}}\right|$ & $\lambda_{2}$ \\
\hline-2.0 & $5.53(\mathbf{0 . 3 9})$ & $1.88(\mathbf{0 . 2 6})$ & 1.88 & $5.97(\mathbf{0 . 5 4})$ & $-2.05(\mathbf{0 . 2 6})$ \\
-4.0 & $7.54(\mathbf{0 . 3 6})$ & $2.69(\mathbf{0 . 3 6})$ & 2.69 & $7.42(\mathbf{0 . 7 4})$ & $-3.93(\mathbf{0 . 3 6})$ \\
-8.0 & $10.45(\mathbf{0 . 4 6})$ & $3.93(\mathbf{0 . 5 6})$ & 3.93 & $10.12(\mathbf{1 . 0 7})$ & $-7.40(\mathbf{0 . 5 4})$ \\
-16.0 & $14.89(\mathbf{0 . 6 4})$ & $5.82(\mathbf{0 . 8 3})$ & 5.82 & $14.45(\mathbf{1 . 7 8})$ & $-15.53(\mathbf{0 . 7 7})$ \\
-32.0 & $20.72(\mathbf{0 . 9 2})$ & $7.96(\mathbf{1 . 5 2})$ & 7.96 & $19.03(\mathbf{3 . 1 1})$ & $-29.92(\mathbf{1 . 4 7})$ \\
\hline
\end{tabular}

The results are shown in Figs. 9 and 10. In the former, five increasing values of threshold are chosen to study the structures identified by the $\lambda_{2}$-method. Next to the surfaces by $\lambda_{2}$, we plot isosurfaces by the magnitude of $\boldsymbol{\omega}_{\mathrm{SAVG}}$ and by the magnitude of average corotation $\boldsymbol{\omega}_{\mathrm{RAVG}}$. The thresholds for these methods were determined again by the volume-matching procedure minimizing the $V_{N} / V_{O}$ ratio.

As a benchmark for the objectivity of the used matching procedure, we perform also a 'dual' matching, based on the previously determined values of $\left|\boldsymbol{\omega}_{\mathrm{RAVG}}\right|$. Note, that the matching procedure is not precisely symmetric in the sense that we would recover the original thresholds of $\lambda_{2}$ in this way. The results of this experiment are presented in Fig. 10.

Table 2 summarizes the absolute thresholds and, more importantly, the $V_{N} / V_{O}$ ratios achieved by the matching procedure used for generation of Figs. 9 and 10. While it is natural to match vortex identification methods, i.e. $\boldsymbol{\omega}_{\mathrm{RAVG}}$ and $\lambda_{2}$, we have decided to include also the average-shear approach into the comparison through this matching procedure. The observation that $\left|\boldsymbol{\omega}_{\mathrm{SAVG}}\right|$ can, for certain thresholds, detect similar regions as vortex identification criteria, including reasonable $V_{N} / V_{O}$ ratios, reflects the fact, that a lot of vorticity in the vortex structures corresponds to shearing motion of its inner layers.

Rather than focusing on horizontal comparisons only, one should view Figs. 9 and 10 also in vertical direction to observe the trends for the individual methods with increasing threshold. Perhaps the most interesting zone in this respect is just behind the sharp tip of the wing, where high shear zone is expected. It can be noticed, that a large portion of this zone is not present in the structure identified by averagecorotation method, even for the lowest threshold. We can also observe, that with an increasing threshold, the structures by $\left|\boldsymbol{\omega}_{\mathrm{SAVG}}\right|$, which correspond to high shear zones, are closer to structures by $\lambda_{2}$-method than to those by $\left|\boldsymbol{\omega}_{\mathrm{RAVG}}\right|$. This can be concluded not only from visual appearance in Figs. 9 and 10, but also quantified by the value of the smallest achieved relative non-overlap $V_{N} / V_{O}$ in Table 2.

We can summarize the results of this experiment so that the structures identified by $\lambda_{2}$-method are matching well the results by average-corotation method farther from the wing, while they tend to incorporate some of the shear-based vorticity near the edges of the wing, independently of the selected threshold. With increasing threshold, the achievable match of $\left|\boldsymbol{\omega}_{\mathrm{SAVG}}\right|$ to $\lambda_{2}$ is much better than that of $\left|\boldsymbol{\omega}_{\mathrm{SAVG}}\right|$ to $\left|\boldsymbol{\omega}_{\mathrm{RAVG}}\right|$ (Table 2), and the structures by $\lambda_{2}$ tend to resemble those by $\left|\boldsymbol{\omega}_{\mathrm{SAVG}}\right|$ also visually. It should be recalled though, that the physical threshold for $\lambda_{2}$-method is $\lambda_{2}=0$, and that the method is not meant to be used with high thresholds. However, the isosurface for $\lambda_{2}=0$ is so complex for this problem, that the vizualization tool (Tecplot 360 2010) was not able to construct it on the given mesh, and we start from the lowest value found to give meaningful results in Fig. 9 and Table 2.

\section{Discussion on Qualitative Features}

Recall some qualitative features of the average-corotation scheme in the context of well-established local methods $Q, \Delta, \lambda_{2}$, and $\lambda_{c i}$ (see Appendix A). All of the given methods are based on the analysis of $\nabla \mathbf{u}$. A mutual geometrical configuration of symmetric and antisymmetric parts of $\nabla \mathbf{u}$ is schematically depicted in Fig. 11 (the ellipsoid is just one chosen representation of strain-rate quadrics).

Let us assume that all the length magnitudes in Fig. 11 are fixed while the vorticity vector can arbitrarily rotate with respect to the ellipsoid given by principal axes of the strain-rate tensor and its eigenvalues. The $Q$-criterion representing the balance between vorticity magnitude and the strain-rate magnitude is clearly $(\nabla \mathbf{u})$-configuration-independent, i.e. it gives the same value regardless of the orientation of the 


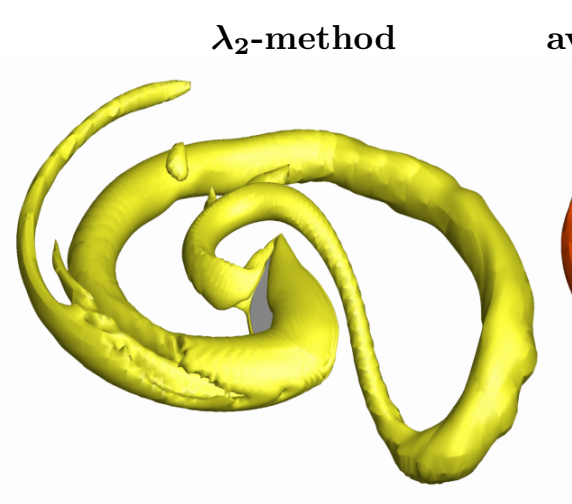

average shear vorticity
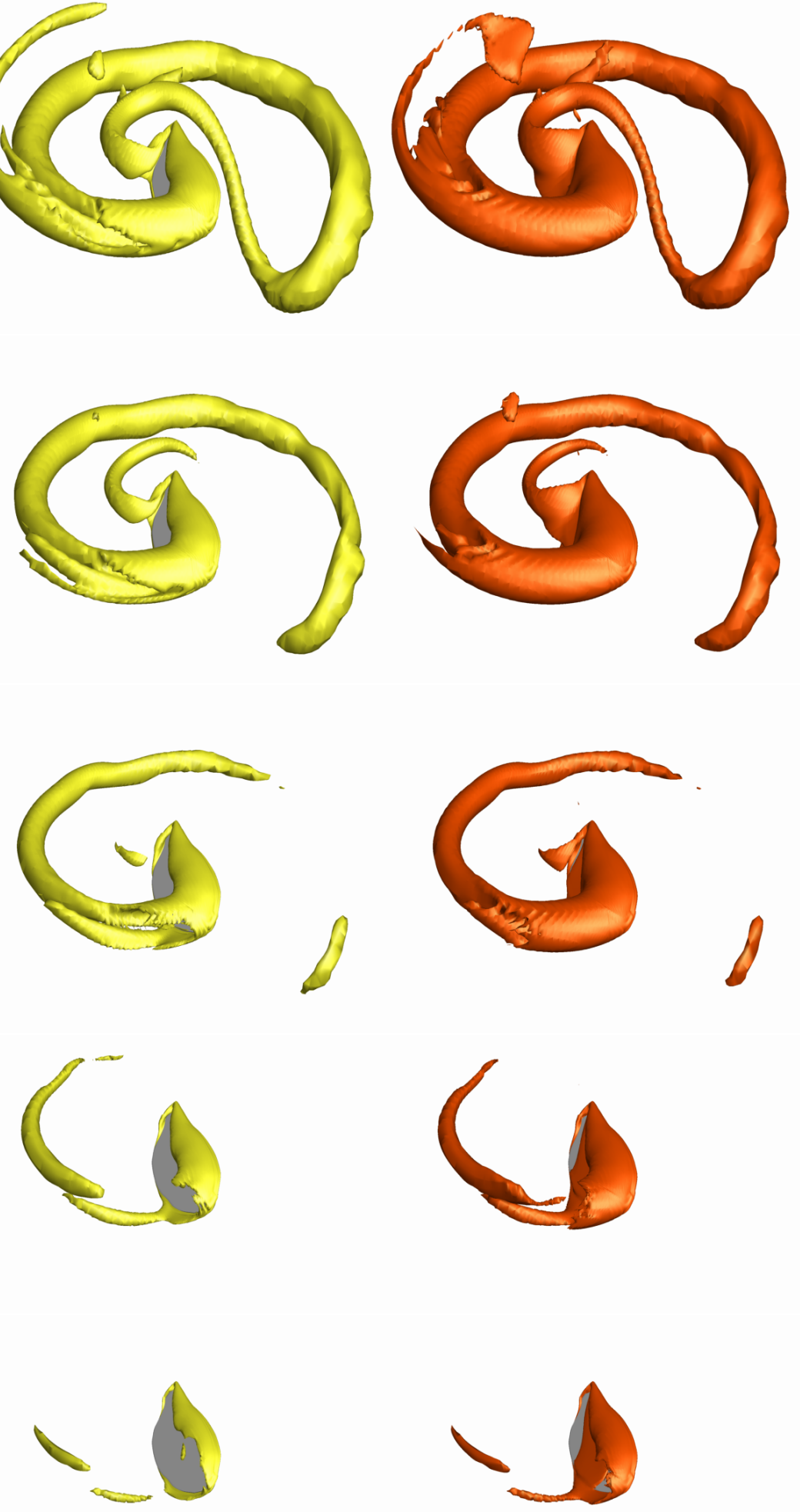
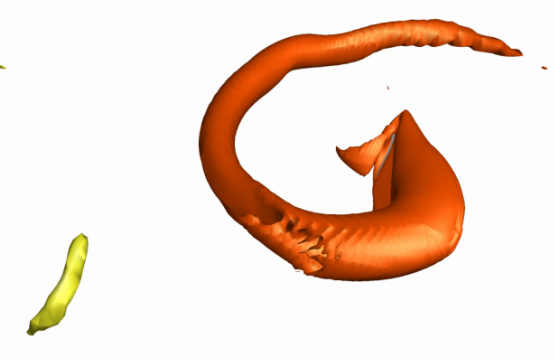

8
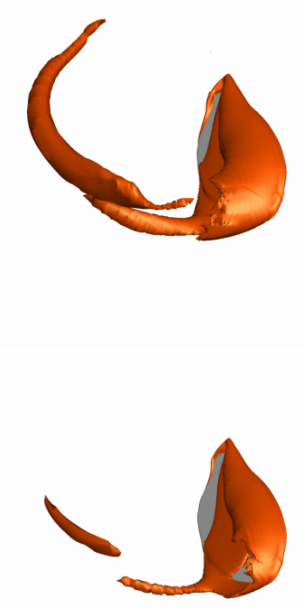

average corotation
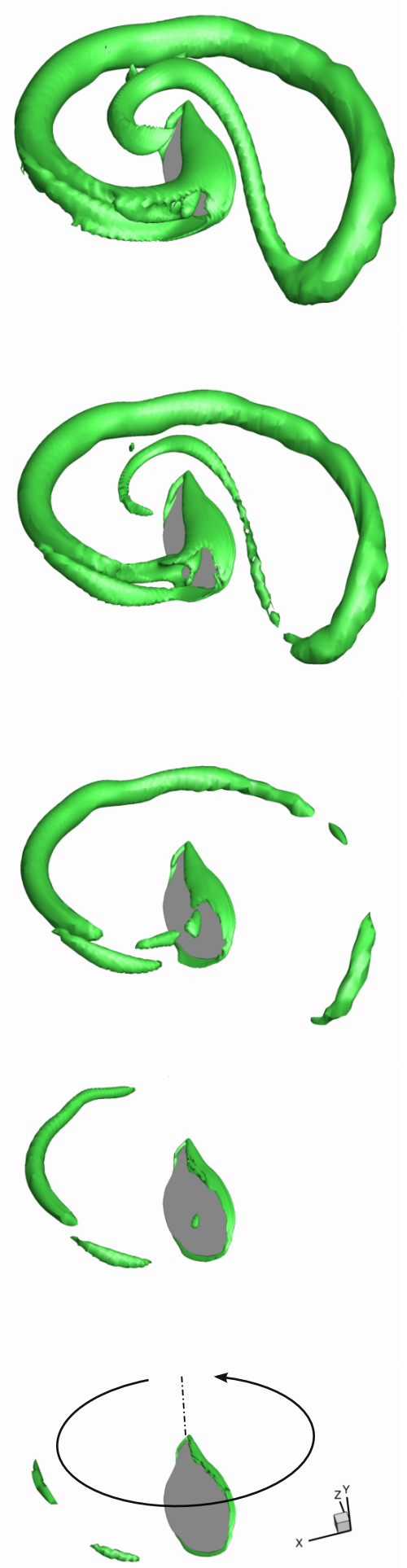

Figure 9: Results for the flow around a revolving (propeller-like motion around $y$-axis) model of a wing of Drosophila at an angle of attack of $40^{\circ}$ and $\mathrm{Re}=500$. Vortex structure for increasing threshold identified by $\lambda_{2}$-criterion (left, threshold: $0.04 \%, 0.08 \%, 0.17 \%, 0.34 \%, 0.67 \%$ ), by magnitude of average shear vorticity $\left|\boldsymbol{\omega}_{\mathrm{SAVG}}\right|$ (center, threshold: $2.0 \%, 2.7 \%, 3.7 \%, 5.3 \%, 7.4 \%$ ), and by average-corotation method (right, threshold: $1.5 \%, 2.1 \%, 3.1 \%, 4.5 \%, 6.2 \%)$. 

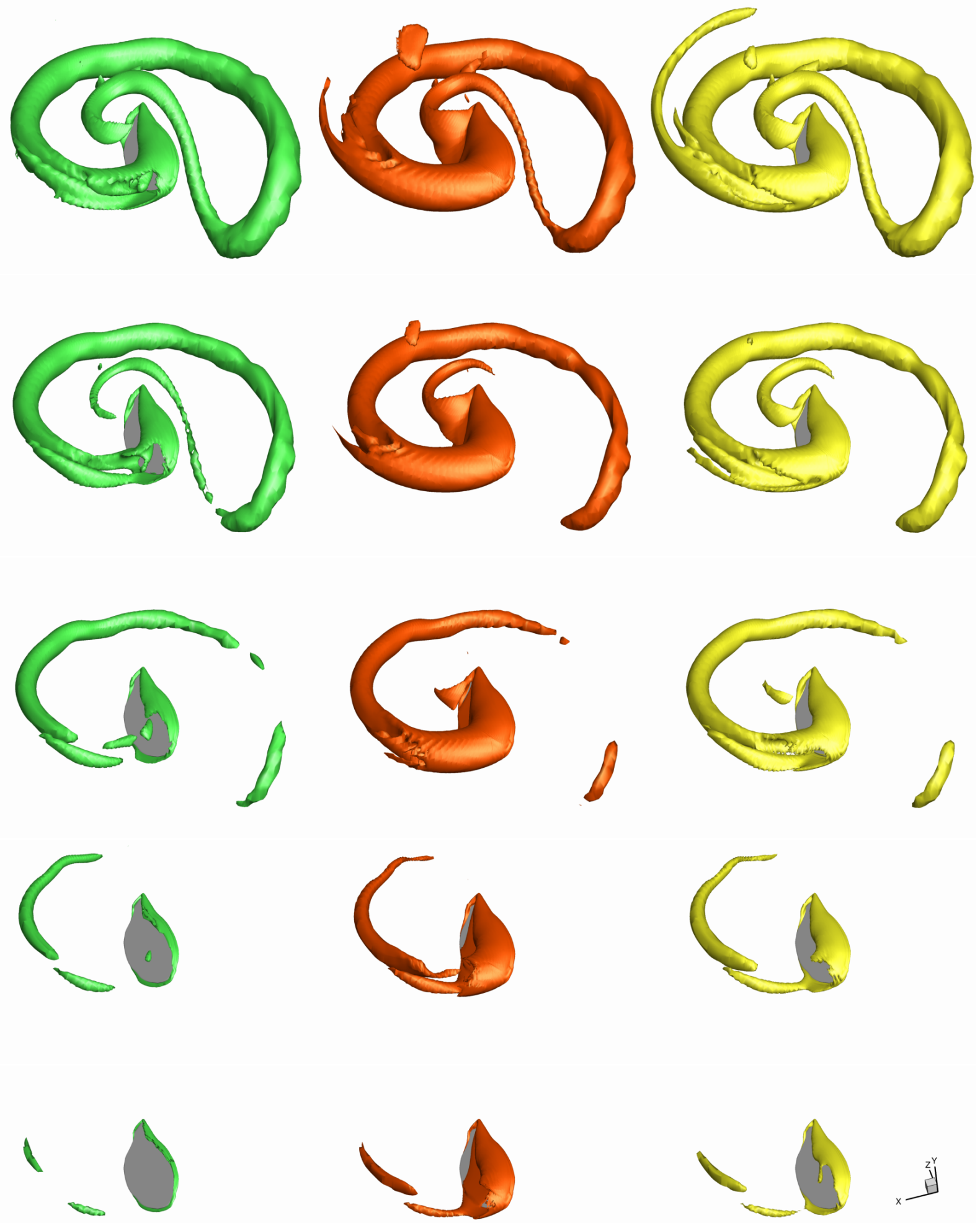

Figure 10: Results for the flow around a revolving (propeller-like motion around $y$-axis) model of a wing of Drosophila at an angle of attack of $40^{\circ}$ and $\mathrm{Re}=500$. Vortex structure for increasing threshold identified by average-corotation method (left, threshold: $1.5 \%, 2.1 \%, 3.1 \%, 4.5 \%, 6.2 \%$ ), by magnitude of average shear vorticity $\left|\boldsymbol{\omega}_{\mathrm{SAVG}}\right|$ (center, threshold: $2.1 \%, 2.7 \%, 3.6 \%, 5.2 \%, 6.8 \%$ ), and by $\lambda_{2}$-criterion (right, threshold: $0.04 \%, 0.08 \%, 0.16 \%, 0.33 \%, 0.63 \%)$. 


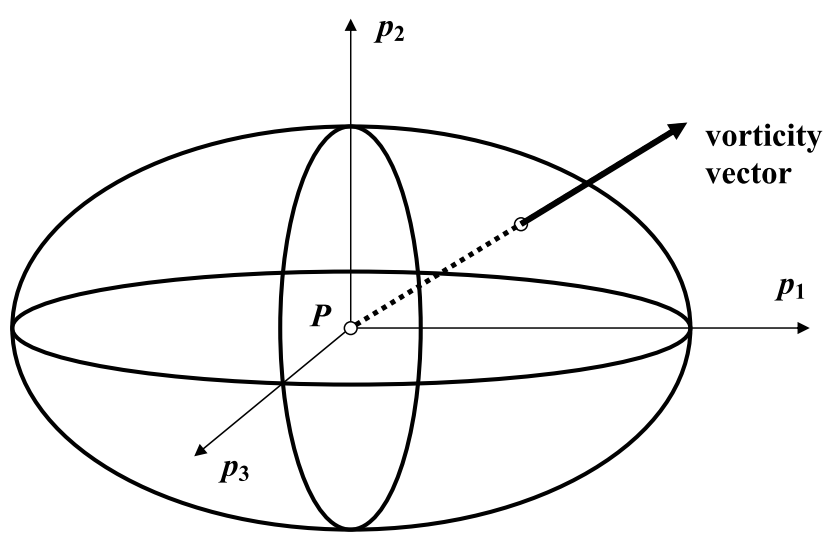

Figure 11: Geometrical configuration of the symmetric part $\left(p_{1}, p_{2}\right.$, and $p_{3}$ are strain-rate principal axes) and antisymmetric part (depicted in terms of vorticity vector) of the velocity-gradient tensor $\nabla \mathbf{u}$.

vorticity vector with respect to the quadric. This fact somewhat lowers its physical value. For example, this insensitivity results in the fact that the following two local flow situations are-from the vortex-identification viewpoint in terms of $Q$-criterion - evaluated as identical, i.e. the value of $Q$ is the same for both cases

$$
\left(\begin{array}{ccc}
-a & 0 & b \\
0 & 2 a & 0 \\
-b & 0 & -a
\end{array}\right) \text { vs. }\left(\begin{array}{ccc}
-a & b & 0 \\
-b & 2 a & 0 \\
0 & 0 & -a
\end{array}\right)
$$

where $a$ and $b$ are positive real numbers. In the first case of local uniaxial isochoric stretching (coupled with uniform radial contraction), the vorticity vector is aligned with the stretching axis, while in the second case of the same stretching situation the vorticity vector is aligned with one of the contracting axes in the plane perpendicular to the stretching axis. As expected, the values of the average-corotation magnitude $\left|\boldsymbol{\omega}_{\mathrm{RAVG}}\right|$ and the swirling strength $\lambda_{c i}$ are for these two different configurations of $\nabla \mathbf{u}$ significantly different in general (depending on the choice of values $a$ and $b$ in (19)). Both these measures of local vortex intensity (or swirling rate) are, similarly as the quantities $\Delta$ and $\lambda_{2},(\nabla \mathbf{u})$-configuration-dependent and hence physically more sound.

Unlike the $Q$-criterion and $\Delta$-criterion (inferred from the invariants of $\nabla \mathbf{u}$ ) and the $\lambda_{2}$-criterion (formulated on dynamic considerations), the measures $\lambda_{c i}$ and $\boldsymbol{\omega}_{\text {RAVG }}$ possess a clear kinematic interpretation of the local swirling motion. Moreover, the criteria $\lambda_{c i}$ and $\boldsymbol{\omega}_{\mathrm{RAVG}}$ are, unlike the schemes $Q, \Delta$, and $\lambda_{2}$, potentially able to determine a representative 'plane of swirling' due to their inherent well-defined flow kinematics (although not employed in the present paper for $\boldsymbol{\omega}_{\mathrm{RAVG}}$ ).

Let us recall the interesting yet controversial idea of the vortex-identification requirement of allowance for an arbitrary axial strain rate. In [26], an analytical diagnosis of four local region-type vortex-identification criteria is performed, demonstrated on the Burgers and Sullivan vortices. It indicates that the $Q$-criterion and $\lambda_{2}$-criterion may cut a connected vortex into broken segments at locations with strong axial stretching. The authors emphasize the following vortex-identification requirements: a generally applicable vortex definition should be able to identify the vortex axis and allow for an arbitrary axial strain. The swirling-strength $\lambda_{c i}$-criterion based on the $\Delta$-criterion, was further enhanced in [12]. The allowance for an arbitrary axial strain rate from [26] became a subject of an intensive debate in [36, 37], as this requirement basically does not conform to the orbital compactness proposed in [12]. For an incompressible flow, the axial strain rate is directly related to the spiraling compactness [12,36]. According to [12, 36], the spiraling compactness requires an appropriate threshold dictated by the length and time scales of the given problem for vortexidentification purpose. Following [37], however, adding a threshold value to the local axial strain rate or to the orbital compactness is subjective and cannot be rationalized. A recent discussion of this issue for the problem of a transitional boundary layer can be found in [38].

According to the previous paragraph, another aspect which is worth paying attention is the stretching sensitivity of the swirling rate, that is, the sensitivity of the local swirling rate to the local stretching rate in the direction perpendicular to the 'plane of swirling'. In the following, the first tensor structure of (19) is to 


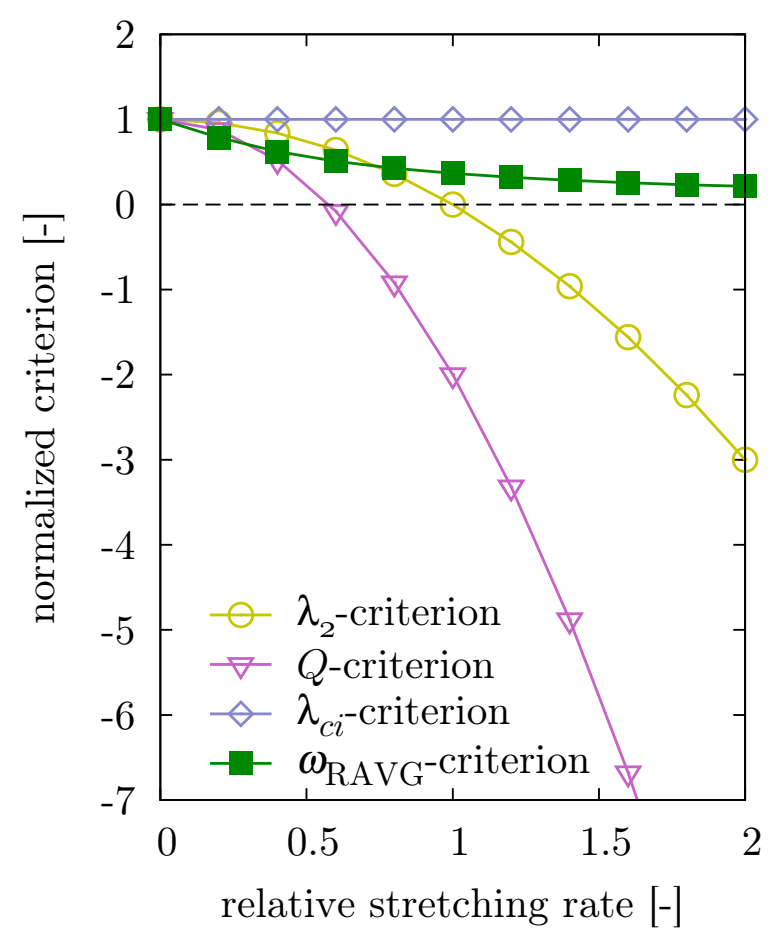

Figure 12: Dependence of various vortex identification criteria on relative stretching rate. Positive values correspond to vortex region.

be analyzed for a fixed value of $b$ and a wide range of $a$ in terms of different vortex-identification schemes. The results for the criteria $\lambda_{2}, Q, \lambda_{c i}$ and $\boldsymbol{\omega}_{\mathrm{RAVG}}$ are shown in Fig. 12 as functions of the relative stretching rate $a / b$. All the results in Fig. 12 are normalized by the corresponding value for the case of zero stretching rate, and only positive values identify a vortex region.

For increasing stretching intensity, the criteria $Q$ and $\lambda_{2}$ soon reach negative values indicating that the examined point does not qualify as a vortex (as usual, the $Q$-criterion is more strict than $\lambda_{2}$-criterion as shown in [6]). On the other hand, the $\lambda_{c i}$-criterion (which is based on the $\Delta$-criterion) is not stretching-sensitive and allows, similarly as the $Q_{2 \mathrm{D}}$-criterion [26] or the maximum-corotation scheme [30], for an arbitrary axial strain rate. In [12] an enhancement of the $\lambda_{c i}$-criterion is introduced, namely a two-parameter scheme for vortex identification, with the second parameter dealing with spiraling compactness of material orbits. As a result, two or three positive thresholds are applied, one for the swirling rate $\lambda_{c i}$ to be above a certain minimum value, and the other one or two to restrict a relative axial-stretching rate (which may be positive for outward spiraling and negative for inward spiraling). Figure 12 indicates that the proposed swirling intensity $\boldsymbol{\omega}_{\mathrm{RAVG}}$ is stretching sensitive, tending asymptotically to zero value for higher stretching rates. Hence, the quantity $\boldsymbol{\omega}_{\mathrm{RAVG}}$ provides a one-parameter stretching-sensitive vortex intensity. However, similarly as the schemes $Q, \Delta$, and $\lambda_{2}$, it does not distinguish between inward and outward spiraling motion what can be determined by the two-parameter scheme [12].

Using the magnitude of the average-corotation vector for vortex identification in general 3D rotational flows requires a positive (yet low) threshold. The physical reason is as follows: in general 3D rotational flows (with the exception of degenerate cases of 2D nature), there exists at least one plane (or a limited bunch of planes) going through the given point, in which non-zero corotation can be detected. However, while almost all planes going through the examined point exhibit non-zero conventional vorticity in a typical non-vortex region, the residual vorticity turns out to be zero at almost all planes. Consequently, a low threshold applied to $\left|\boldsymbol{\omega}_{\mathrm{RAVG}}\right|$ is sufficient to suppress such regions in vortex visualization. In addition, recall that for visualization purposes, a non-zero threshold is often employed also for the widely used local criteria, as the vortex surface with a positive threshold appears significantly smoother [9].

Finally, let us mention the issue of smoothness of vortical structures identified by the maximum-corotation approach [30]. From the spatial viewpoint, the plane of maximum corotation may change abruptly, or it 

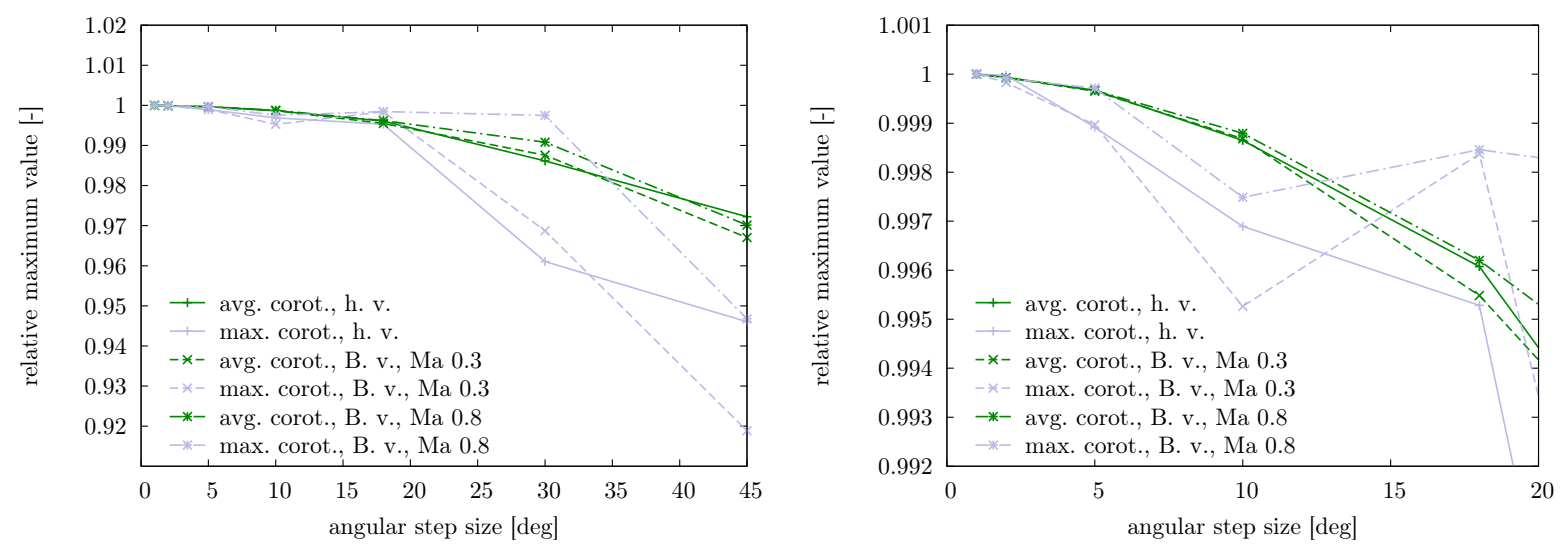

Figure 13: Convergence analysis: Comparison of relative maximum values for both average- and maximumcorotation criteria normalized by the maximum values for one-degree resolution; for angular step 1-45 degrees (left), and detail for 1-20 degrees (right), 'h. v.' stands for a hairpin vortex, 'B. v.' for Burgers vortices.

may become even ambiguous for some velocity gradient data, hence its normal direction necessary for vector representation of the residual vorticity $\omega_{\text {RES }}$ according to (11) may change abruptly as well. As can be seen in Fig. 3, this may lead to a reasonable agreement of maximum-corotation scheme with the other methods only for higher thresholds. In contrast to the maximum corotation, the averaging procedure enhances the pointwise stability of the average corotation $\boldsymbol{\omega}_{\mathrm{RAVG}}$, which in turn leads to significantly smoother surfaces of identified vortical structures. In addition, average corotation provides a unique vector for any input data. For this reason, it is better suited for visualization purposes.

\section{Discussion on Numerical Precision}

The average corotation $\boldsymbol{\omega}_{\mathrm{RAVG}}$, according to formula (12), is evaluated by means of numerical integration. Therefore, a new important aspect emerges, namely the sensitivity to the numerical precision of the quadrature formula.

Let us now analyze the precision of the simple rectangle quadrature (16). Discretizing the domain $[0,2 \pi] \times[0, \pi]$ into squares with angular step size $\delta$, the dependence of $\boldsymbol{\omega}_{\text {RAVG }}$ on the discretization parameter $\delta$ is investigated. The same sampling is also employed for calculating the maximum corotation for comparison.

The $\boldsymbol{\omega}_{\mathrm{RAVG}}$-method behaves as a robust scheme while increasing the angular step starting with the finest (here $\delta=\pi / 180=1^{\circ}$ ) resolution. Figure 13 shows maximum values of $\left|\boldsymbol{\omega}_{\mathrm{RAVG}}\right|$, found over the whole domain and normalized by the maximum values for one-degree resolution, using different angular step sizes. For comparison purposes the maximum-corotation results are included. The six curves in Fig. 13 illustrate the two corotation methods for three flow situations from Section 4. In the right part of Fig. 13, a more detailed view focussing on the region up to the angular step of $20^{\circ}$ is shown. Figure 13 confirms the convergence behavior for a decreasing angular step.

Figure 14 depicts normalized maximum differences found over the whole domain, that is, by examining point by point the difference between 'one-degree' values and lower-resolution values, normalizing each difference by the overall maximum found for the finest one-degree resolution. As before, the right part of Fig. 14 provides a more detailed view up to the angular step of $20^{\circ}$. For the angular step $20^{\circ}$ and less, the overall relative maximum difference is less (or much less) than one percent for all examined flow situations.

The following conclusion can be drawn: the average-corotation scheme tends to converge smoothly in a very fast and stable manner. This is not surprising as (16) represents a numerical integration method with first-order accuracy in each dimension. When a uniform refinement of the step size is performed in both angular coordinates, we have a scheme with quadratic order of convergence to the exact value of the integral (15), as can be clearly seen in Figs. 13-14. In addition, the robustness of the average-corotation method in the case of a hairpin vortex is illustrated in Fig. 15 in terms of isosurfaces determined at a fixed threshold using different angular step sizes.

Summing up, the observed robustness is very good and allows the use of a relatively large step size. 

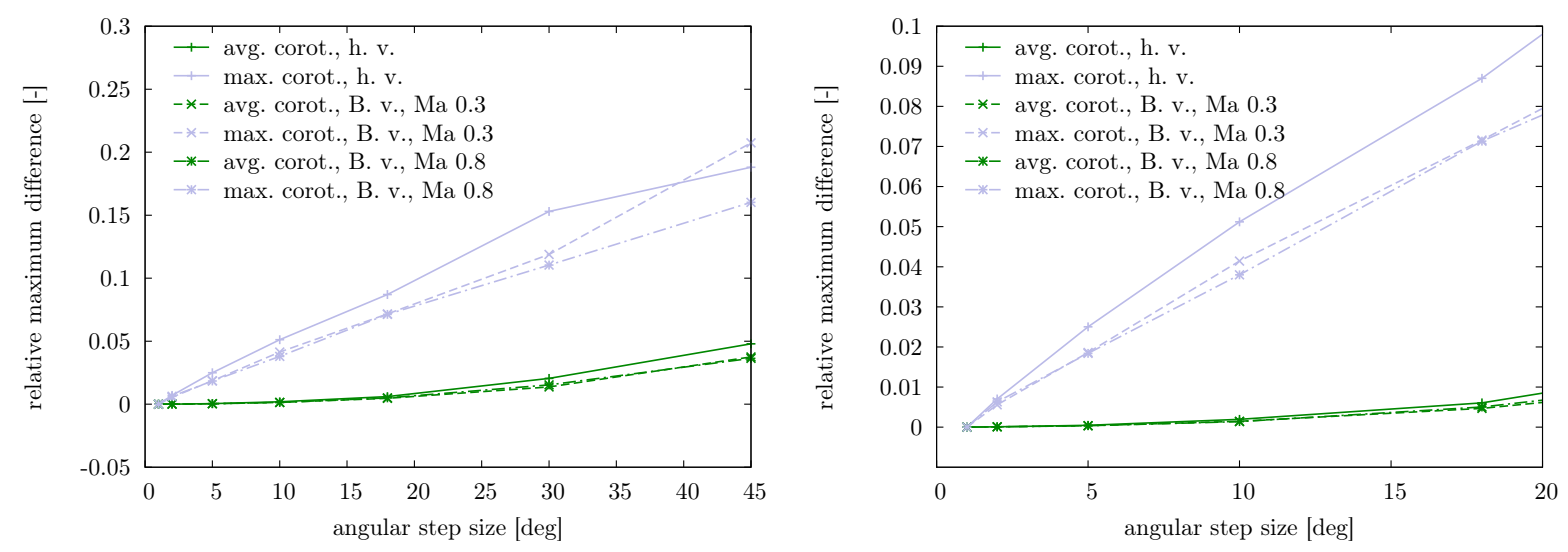

Figure 14: Normalized difference with respect to value for angular step size 1 degree: maximum values over the whole domain for both average and maximum-corotation criteria normalized by the maximum values for one-degree resolution; for angular step 1-45 degrees (left), and detail for 1-20 degrees (right), 'h. v.' stands for a hairpin vortex, 'B. v.' for Burgers vortices.

For example, the angular step size of $10^{\circ}$ appears to be sufficient for practical computations. This may be important, because the time needed for numerical integration using the finest tested resolution (one degree) may be considerably larger than for the standard methods. However, in our experience, already using an angular step size of $5^{\circ}$ was found to present only little overhead compared to other parts of a flow field solution. To provide some idea on computing times, on an Intel Core Duo CPU with $2.53 \mathrm{GHz}$, the computing time per one data point was 0.076 milliseconds for the angular step size $10^{\circ}$, while for step size $1^{\circ}, 7.714$ milliseconds were needed. This agrees well with the expected scaling, since going from $10^{\circ}$ to $1^{\circ}$, the computation requires $10^{2}$ times more evaluations inside the sum of (16), making in turn the evaluation proportionally more expensive.

In comparison, using Fibonacci integration from [32] with 752 integration points led to accuracy comparable to rectangle rule with $1^{\circ}$ resolution (i.e. 64,800 points), requiring only 0.2 milliseconds for each point. Based on our experience, this method can be recommended for accelerating the evaluation of the average corotation. On the other hand, while being even faster, the scheme from [31] using only 122 points did not provide sufficient accuracy for our problems.

\section{Conclusions}

An easy-to-interpret kinematic quantity of local vortex intensity is introduced. The measure is pointwise and based on the velocity-gradient tensor $\nabla \mathbf{u}$ like many popular local methods $\left(Q, \Delta, \lambda_{2}\right.$, and $\left.\lambda_{c i}\right)$. The proposed quantity, the average corotation of material line segments near a point $\boldsymbol{\omega}_{\text {RAVG }}$ given by (12), is a vector obtained by averaging the instantaneous local rigid-body rotation over 'all planar cross-sections' going through the considered point. On a selected plane, the rigid-body rotation is quantified by residual vorticity obtained after the elimination of shearing motion near a point on the given plane. At points with zero strain rate, the average corotation just reproduces the vector of conventional vorticity. At points where strain rate is present, the vector of average corotation represents the portion of vorticity that can be attributed to rigid-body rotation.

Practical applications of $\boldsymbol{\omega}_{\mathrm{RAVG}}$ in vortex identification for a hairpin vortex of boundary-layer transition, the reconnection process of two Burgers vortices, a flow around a flat plate at an angle of attack of $30^{\circ}$, and a flow around a revolving insect wing are presented. The $\boldsymbol{\omega}_{\mathrm{RAVG}}$-criterion provides a high level of agreement with the $\lambda_{2}$-criterion. However, there is one positive difference: the shearing bias - found for the $\lambda_{2}$-criterion and $Q$-criterion which interpret the shearing zones around the plate and wing edges as vortex zones-has been eliminated.

From the comparison with the schemes $Q, \Delta, \lambda_{2}$, and $\lambda_{c i}$, some useful qualitative features of $\boldsymbol{\omega}_{\mathrm{RAVG}}$ can be deduced. The proposed measure possesses both a clear kinematic interpretation and stretching sensitivity, in contrast to the criteria $Q, \Delta, \lambda_{2}$, and $\lambda_{c i}$ satisfying only one of these aspects. Unlike the $Q$-criterion, $\boldsymbol{\omega}_{\mathrm{RAVG}}$ 
$\lambda_{2}$-method (2.6\%)
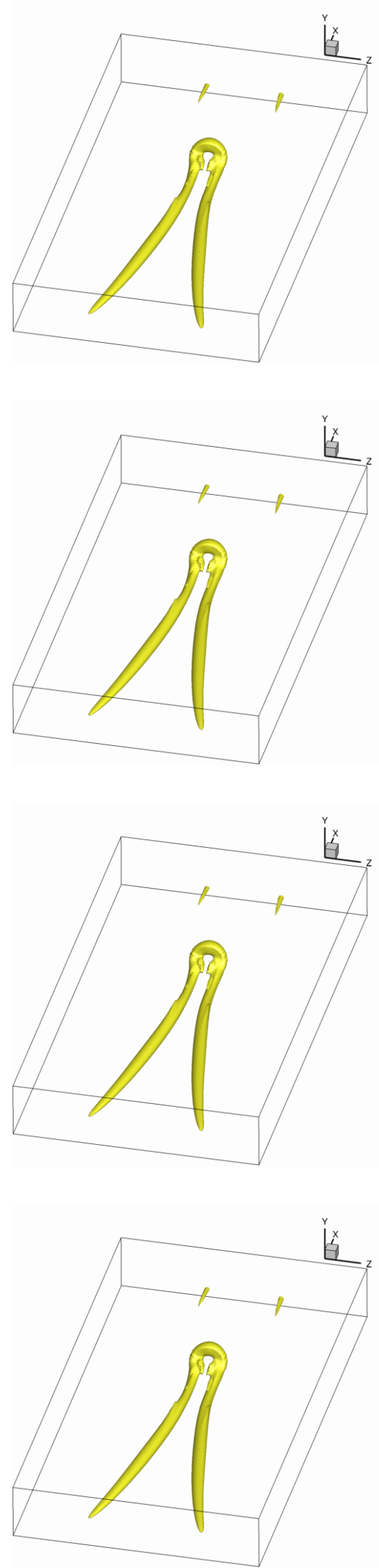

average corot. $(\mathbf{1 0 . 0 \% )}$

angular step of $1^{\circ}$

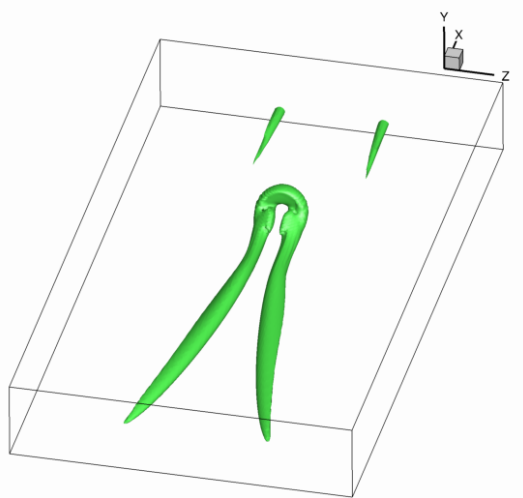

angular step of $18^{\circ}$

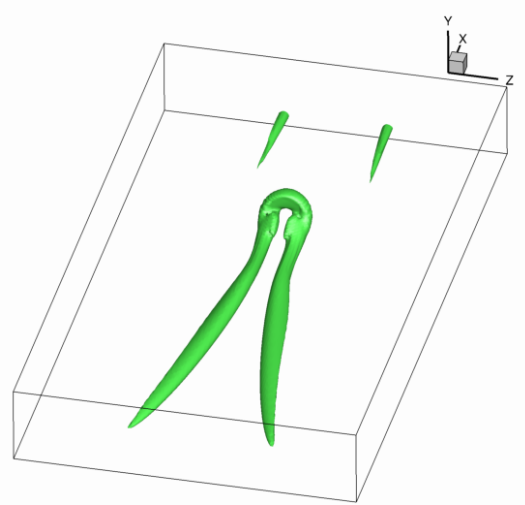

angular step of $45^{\circ}$
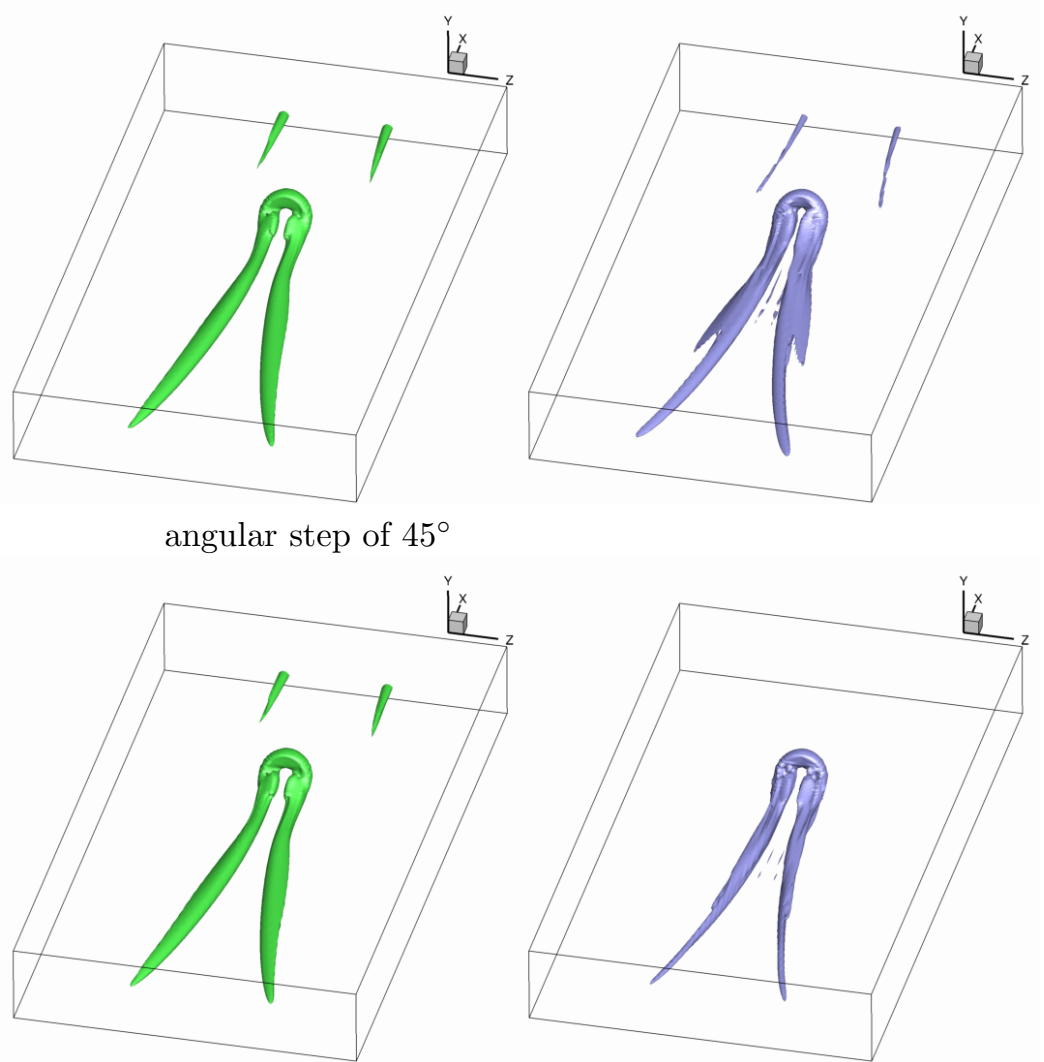

angular step of $90^{\circ}$
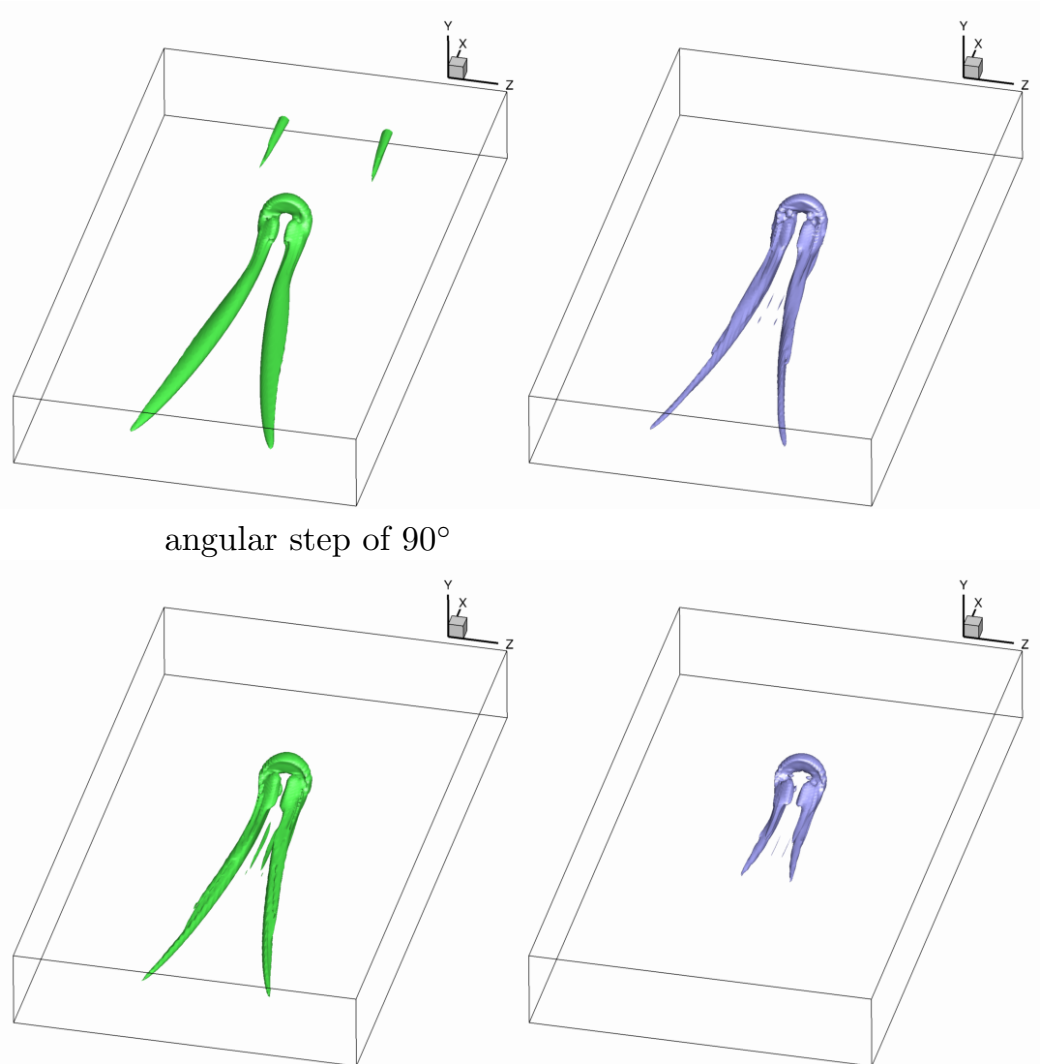

maximum corot. $(\mathbf{1 6 . 8 \% )}$

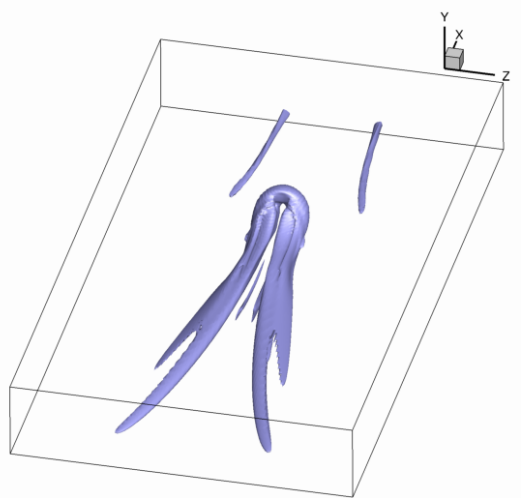

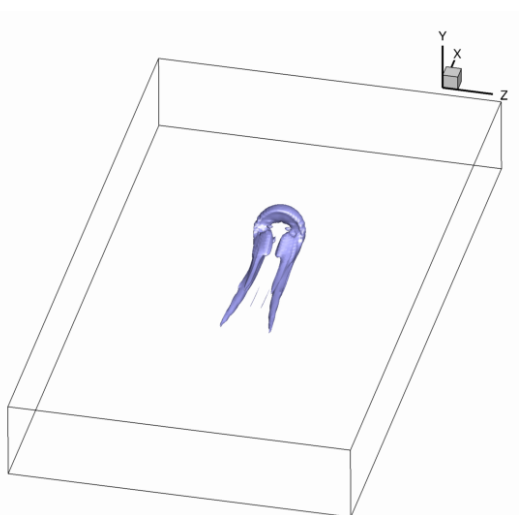

Figure 15: Dependence of results on the angular step size for both average- and maximum-corotation methods at fixed thresholds (shown in parentheses), the $\lambda_{2}$-criterion is presented for comparison purposes and repeated on each row of the plot. 
is sensitive to the $(\nabla \mathbf{u})$-configuration, and unlike the $Q, \Delta$, and $\lambda_{2}$ schemes, the $\boldsymbol{\omega}_{\mathrm{RAVG}}$-scheme is, similarly as the $\lambda_{c i}$-criterion, potentially able to determine the 'plane of swirling' due to its vector nature.

It should be noted that the widely used $Q, \Delta, \lambda_{2}$, and $\lambda_{c i}$ schemes need only one analytical evaluation, while the $\boldsymbol{\omega}_{\mathrm{RAVG}}$-criterion needs numerical integration with a chosen precision. The precision of the approximated value of $\boldsymbol{\omega}_{\mathrm{RAVG}}$ behaves in a predictable manner. This feature is lacking in the previous maximum-corotation scheme [30] of similar complexity as well as in the earlier triple-decomposition method [15] of even higher complexity. Consequently, an angular step of $5^{\circ}$ or $10^{\circ}$ can be safely recommended for providing a feasible compromise between accuracy and required computational time of the numerical integration. In addition, advanced techniques for numerical integration on a sphere, such as Fibonacci numerical integration [32], can be employed to accelerate evaluation of the average corotation.

As a well-defined kinematic approach, the proposed average-corotation method is readily applicable to compressible and variable-density flows in contrast to the $\lambda_{2}$-criterion. Recall, that the $\Delta$-criterion and the associated $\lambda_{c i}$-criterion are directly extendable to compressible flows, while the $Q$-criterion needs to be $a$ priori redefined in terms of deviatoric strain rate to be applicable to compressible flows [39].

\section{Acknowledgements}

The authors are very grateful to Prof. Ulrich Rist and Dr. Kudret Baysal, IAG, University of Stuttgart, for providing some of the DNS data sets used in the present paper. This work was supported by the Grant Agency of the Academy of Sciences of the Czech Republic through grant IAA200600801, by Ministry of Education, Youth and Sports of the Czech Republic under research project LH11004, and by the Academy of Sciences of the Czech Republic through RVO:67985874 and RVO:67985840.

\section{A Vortex Identification Methods}

A brief survey of vortex-identification schemes is presented in Tables 3 and 4, note that this is far from being a complete list.

$\boldsymbol{Q}$-criterion [3]: Vortices of an incompressible flow are identified as connected fluid regions with a positive second invariant of the velocity-gradient tensor $\nabla \mathbf{u}, \nabla \mathbf{u}=\mathbf{S}+\boldsymbol{\Omega}, \mathbf{S}$ is the strain-rate tensor, $\boldsymbol{\Omega}$ is the vorticity tensor (in tensor notation below the subscript comma denotes differentiation),

$$
Q \equiv \frac{1}{2}\left(u_{i, i}^{2}-u_{i, j} u_{j, i}\right)=-\frac{1}{2} u_{i, j} u_{j, i}=\frac{1}{2}\left(\|\boldsymbol{\Omega}\|^{2}-\|\mathbf{S}\|^{2}\right)>0 .
$$

This is fulfilled in the regions where the vorticity magnitude prevails over the strain-rate magnitude.

$\boldsymbol{\Delta}$-criterion $[1,2,4]$ : Vortices are defined as the regions in which the eigenvalues of $\nabla \mathbf{u}$ are complex and the streamline pattern is spiraling or closed in a local reference frame moving with the point. To guarantee complex eigenvalues of $\nabla \mathbf{u}$ the discriminant $\Delta$ of the characteristic equation should be positive

$$
\Delta=\left(\frac{Q}{3}\right)^{3}+\left(\frac{R}{2}\right)^{2}>0
$$

where $Q$, given by (A.1), and $R \equiv \operatorname{Det}\left(u_{i, j}\right)$ are the second and third invariants of $\nabla \mathbf{u}$, respectively. The $\Delta$-criterion (A.2) is valid for incompressible flows only. Substituting (A.1) into (A.2), one can see that the $Q$-criterion is more restrictive than the $\Delta$-criterion.

$\boldsymbol{\lambda}_{\mathbf{2}}$-criterion [6]: This criterion is formulated on dynamic considerations, namely on the search for a pressure minimum across the vortex. The quantity $\mathbf{S}^{2}+\boldsymbol{\Omega}^{2}$ is employed as an approximation of the pressure Hessian after removing the unsteady irrotational straining and viscous effects from the strain-rate transport equation for incompressible fluids. A vortex region is defined as a connected fluid region with two negative eigenvalues of $\mathbf{S}^{2}+\boldsymbol{\Omega}^{2}$ (that is, if these eigenvalues are ordered, $\lambda_{1} \geq \lambda_{2} \geq \lambda_{3}$, by the condition $\lambda_{2}<0$ ).

$\boldsymbol{\lambda}_{\boldsymbol{c} i}$-criterion [9, 12]: The $\Delta$-criterion has motivated the so-called swirling-strength criterion denoted as $\lambda_{c i}$-criterion based on the imaginary part of the complex conjugate eigenvalues of $\nabla \mathbf{u}$. The time period for 
Table 3: Region-type vortex-identification methods

\begin{tabular}{|c|}
\hline $\begin{array}{l}\text { Author(s) \& Year } \\
\text { Basic characteristics }\end{array}$ \\
\hline Dallmann (1983) [1] \\
\hline$\Delta$-criterion: complex eigenvalues of $\nabla \mathbf{u}$ \\
\hline Vollmers et al. (1983) [2] \\
\hline$\Delta$-criterion: complex eigenvalues of $\nabla \mathbf{u}$ \\
\hline Hunt et al. (1988) [3] \\
\hline$Q$-criterion: second invariant of $\nabla \mathbf{u}$ \\
\hline Chong et al. (1990) $[4]$ \\
\hline$\Delta$-criterion: complex eigenvalues of $\nabla \mathbf{u}$ \\
\hline $\begin{array}{l}\text { Berdahl \& Thompson (1993) }[5] \\
\text { swirl parameter, similar to } \lambda_{c i} \text {-criterion }\end{array}$ \\
\hline Jeong \& Hussain (1995) [6] \\
\hline$\lambda_{2}$-criterion: eigenvalues of $\mathbf{S}^{2}+\boldsymbol{\Omega}^{2}$ \\
\hline $\begin{array}{l}\text { Portela (1997) }[7] \\
\text { scheme based on set theory }\end{array}$ \\
\hline $\begin{array}{l}\text { Cucitore et al. (1999) [8] } \\
\text { non-local particle-trajectory method }\end{array}$ \\
\hline $\begin{array}{l}\text { Zhou et al. (1999) [9] } \\
\text { swirling-strength } \lambda_{c i} \text {-criterion: complex eigenvalues of } \nabla \mathbf{u}\end{array}$ \\
\hline $\begin{array}{l}\text { Sadarjoen \& Post (2000) [10] } \\
\text { advanced streamline method }\end{array}$ \\
\hline $\begin{array}{l}\text { Jiang et al. (2002) [11] } \\
\text { scheme based on combinatorial topology }\end{array}$ \\
\hline $\begin{array}{l}\text { Chakraborty et al. (2005) [12] } \\
\text { enhanced swirling-strength } \lambda_{c i} \text {-criterion }\end{array}$ \\
\hline $\begin{array}{l}\text { Haller (2005) }[13] \\
\text { objective frame-independent vortex definition }\end{array}$ \\
\hline Zhang \& Choudhury (2006) [14] \\
\hline Galilean-invariant eigen helicity density \\
\hline $\begin{array}{l}\text { Koláŕ }(\mathbf{2 0 0 7})[15] \\
\text { triple decomposition of } \nabla \mathbf{u} \text { : residual vor }\end{array}$ \\
\hline
\end{tabular}

Table 4: Line-type vortex-identification methods

\begin{tabular}{|l|}
\hline Author(s) \& Year \\
Basic characteristics \\
\hline $\begin{array}{l}\text { Levy et al. (1990) [16] } \\
\text { extrema of normalized helicity density }\end{array}$ \\
\hline $\begin{array}{l}\text { Banks \& Singer (1995) [17] } \\
\text { vorticity-predictor and pressure-corrector scheme }\end{array}$ \\
\hline $\begin{array}{l}\text { Sujudi \& Haimes (1995) [18] } \\
\text { eigenvectors of } \nabla \mathbf{u}\end{array}$ \\
\hline $\begin{array}{l}\text { Kida \& Miura (1998) [19] } \\
\text { sectional-swirl and pressure-minimum scheme }\end{array}$ \\
\hline $\begin{array}{l}\text { Roth \& Peikert (1998) [20] } \\
\text { parallel-vectors (higher-order) method }\end{array}$ \\
\hline $\begin{array}{l}\text { Strawn et al. (1999) [21] } \\
\text { lines of maximum vorticity }\end{array}$ \\
\hline $\begin{array}{l}\text { Roth (2000) [22] } \\
\text { generalization of earlier line-type methods }\end{array}$ \\
\hline
\end{tabular}


completing one revolution of the streamline on the plane spanned by the complex eigenvectors is given by $2 \pi / \lambda_{c i}$ [12]. The two criteria, $\Delta$ and $\lambda_{c i}$, are equivalent only for zero thresholds $\left(\Delta=0\right.$ and $\left.\lambda_{c i}=0\right)$.

Probably the most detailed study of various line-type methods is conducted in [22]. However, there is a number of other recent papers on this subject, for example [23, 24, 40].

\section{B Two-Dimensional Kinematics and Residual Vorticity}

For the sake of simplicity, let us assume an arbitrary 2D deviatoric (i.e. traceless) velocity-gradient tensor $\nabla \mathbf{u}$, for which $\left(u_{x}+v_{y}\right)=0$. As emphasized in Section 2, only deviatoric motion is decisive in the present corotation analysis. Considering rotation of coordinates $x$ and $y$ around the third axis (or the plane normal), the tensor $\nabla \mathbf{u}$ can be described in an arbitrary reference frame, in the system of principal axes, and in the specific reference frame, originally introduced as a basic reference frame (BRF) in [15], as follows

$$
\left(\begin{array}{cc}
u_{x} & u_{y} \\
v_{x} & -u_{x}
\end{array}\right) \longrightarrow\left(\begin{array}{cc}
s & -\omega \\
\omega & -s
\end{array}\right)^{\text {PRINCIPAL AXES }} \longrightarrow\left(\begin{array}{cc}
0 & s-\omega \\
s+\omega & 0
\end{array}\right)^{\mathrm{BRF}},
$$

where the planar deviatoric principal strain rate $s$ (for simplicity, the subscript ' $D$ ' denoting deviatoric in Section 2 is omitted) and the vorticity tensor component $\omega$ fulfil

$$
\begin{aligned}
|s| & =\left(\sqrt{4 u_{x}^{2}+\left(u_{y}+v_{x}\right)^{2}}\right) / 2, \\
\omega & =\left(v_{x}-u_{y}\right) / 2 .
\end{aligned}
$$

There are two different relative rotational orientations of $u_{y} \mathrm{~d} y$ and $v_{x} \mathrm{~d} x$ in the BRF, the same and the opposite, see Fig. 16. In Fig. 16(a), vorticity dominates over deviatoric strain rate. The characteristic angles $\alpha_{1}$ and $\alpha_{2}$ correspond to the residual vorticity $\omega_{\text {RES }}$ (associated with the rigid-body rotation) and shear vorticity $\omega_{\mathrm{SH}}$ (associated with the pure shearing motion) while their sum is proportional to the total (conventional) vorticity $\omega$. In Fig. 16(b), deviatoric strain rate dominates over vorticity. The characteristic angles $\beta_{1}$ and $\beta_{2}$ correspond to the residual strain rate $s_{\mathrm{RES}}$ and shear strain rate $s_{\mathrm{SH}}$ while their sum is proportional to the total (conventional) strain rate $s$. The same signs of the quantities in couples $\left(\omega_{\mathrm{RES}}, \omega_{\mathrm{SH}}\right)$ and $\left(s_{\mathrm{RES}}, s_{\mathrm{SH}}\right)$ indicate the non-destructive nature of the superimposing construction of the analyzed infinitesimal motion depicted in Fig. 16. For both rotational orientations, the magnitude of the superimposed shearing motion is given by the difference of the absolute values of $u_{y}$ and $v_{x}$. In planar flows, a non-zero $\omega_{\text {RES }}$, apparently existing only for the same rotational orientation of $u_{y} \mathrm{~d} y$ and $v_{x} \mathrm{~d} x$ (see Fig. 16), excludes the existence of a non-zero $s_{\text {RES }}$, which exists only for the opposite rotational orientation of $u_{y} \mathrm{~d} y$ and $v_{x} \mathrm{~d} x$.

With respect to (B.1) showing explicitly the desired velocity-gradient tensor components in the BRF in terms of the 'explicit competition' between $s$ and $\omega$, and assuming $|s| \geq|\omega|$ or $|s| \leq|\omega|$, the following set of relations can be derived for $s$ and $\omega$ and their residual and shear components (note that the limiting case $|s|=|\omega|$ represents a simple shear) [15]

$$
\begin{aligned}
s & =s_{\mathrm{RES}}+s_{\mathrm{SH}}, \\
|s| & =\left|s_{\mathrm{RES}}\right|+\left|s_{\mathrm{SH}}\right|, \\
s_{\mathrm{SH}} & =(\operatorname{sgn} s)|\omega| \text { for }|s| \geq|\omega|, \\
s_{\mathrm{SH}} & =s \text { for }|s| \leq|\omega|, \\
s_{\mathrm{RES}} & =s-s_{\mathrm{SH}}=(\operatorname{sgn} s)[|s|-|\omega|] \text { for }|s| \geq|\omega|, \\
s_{\mathrm{RES}} & =s-s_{\mathrm{SH}}=0 \text { for }|s| \leq|\omega|, \\
\omega & =\omega_{\mathrm{RES}}+\omega_{\mathrm{SH}}, \\
|\omega| & =\left|\omega_{\mathrm{RES}}\right|+\left|\omega_{\mathrm{SH}}\right|, \\
\omega_{\mathrm{SH}} & =\omega \text { for }|s| \geq|\omega|, \\
\omega_{\mathrm{SH}} & =(\operatorname{sgn} \omega)|s| \text { for }|s| \leq|\omega|, \\
\omega_{\mathrm{RES}} & =\omega-\omega_{\mathrm{SH}}=0 \text { for }|s| \geq|\omega|, \\
\omega_{\mathrm{RES}} & =\omega-\omega_{\mathrm{SH}}=(\operatorname{sgn} \omega)[|\omega|-|s|] \text { for }|s| \leq|\omega| .
\end{aligned}
$$




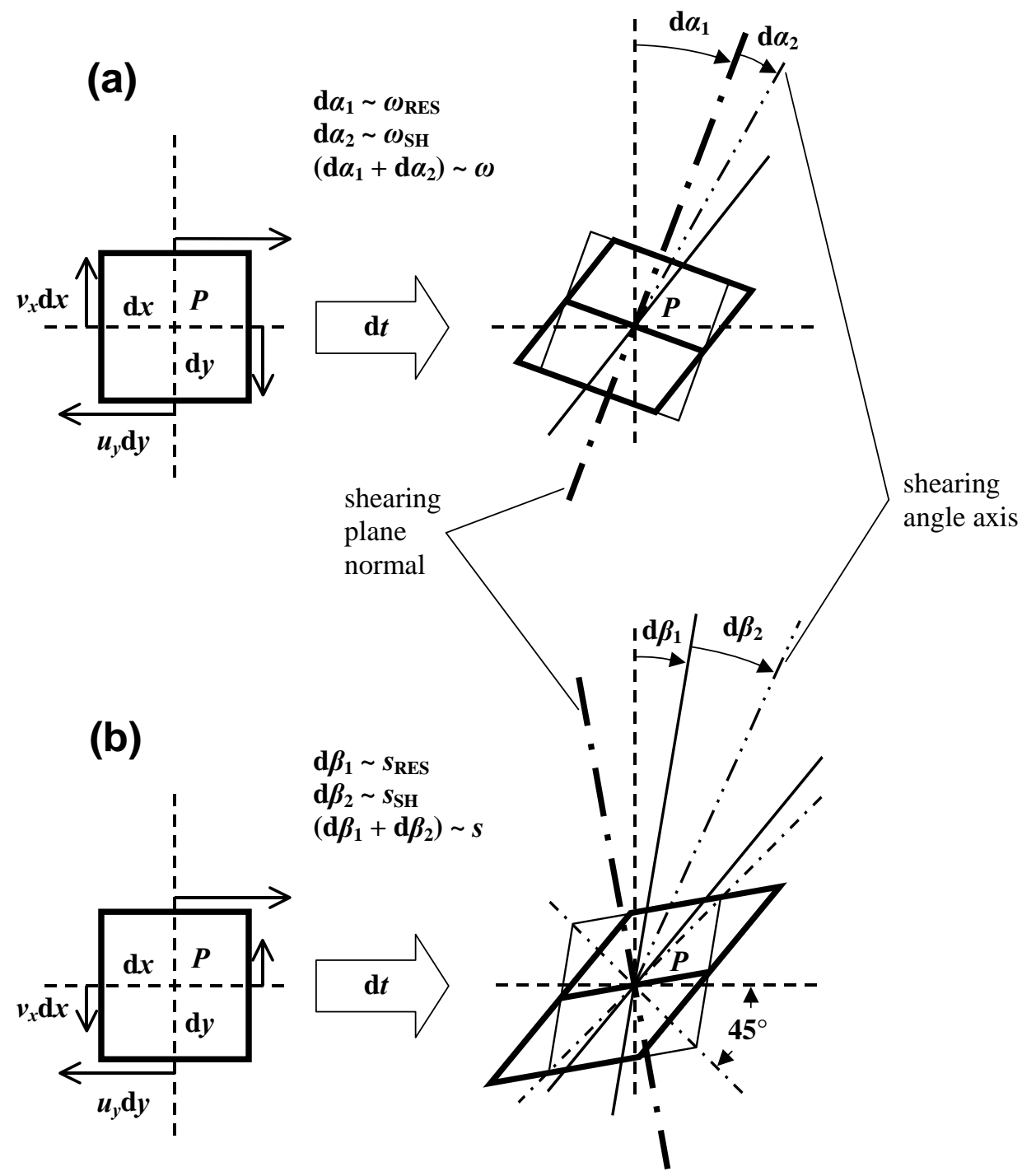

Figure 16: Geometrical interpretation of infinitesimal local relative motion near a point: (a) rigid-body rotation and pure shearing $(|s|<|\omega|)$, (b) irrotational straining and pure shearing $(|s|>|\omega|)$. Reproduced from [15]. 
The following conclusion can be inferred from (B.15): the magnitude of planar residual vorticity represents nothing but the simplest measure of the dominance of the planar vorticity magnitude over the planar strainrate magnitude. Analogously, from (B.8), the magnitude of planar residual strain rate is nothing but the simplest measure of the dominance of the planar strain-rate magnitude over the planar vorticity magnitude.

\section{Determination of the Scaling Factor $\alpha$ in Equation (12)}

Let us determine a well-justified value for the scaling factor $\alpha$ introduced in (12). Its derivation is based on a natural requirement that the conventional vorticity $\boldsymbol{\omega}=\nabla \times \mathbf{u}$, should not be altered by the application of the averaging procedure.

More specifically, let us define the vector of conventional vorticity related to a plane given by $\mathbf{n}$ similarly to formula (11) as

$$
\omega_{\mathbf{n}}\left(\mathbf{x}_{0}, \mathbf{n}\right)=2 \omega \mathbf{n} .
$$

Suppose a given vorticity vector $\boldsymbol{\omega}$, that should be reproduced by averaging the quantity $\boldsymbol{\omega}_{\mathbf{n}}$ in place of $\boldsymbol{\omega}_{\text {RES }}$ in (12). Figure 17 illustrates the derivation. The first important step is to realize, that $\boldsymbol{\omega}_{\mathbf{n}}$ is nothing but a projection of $\boldsymbol{\omega}$ to the direction of $\mathbf{n}$, i.e.

$$
\boldsymbol{\omega}_{\mathbf{n}}=\left(\boldsymbol{\omega}^{T} \mathbf{n}\right) \mathbf{n}=\left(\mathbf{n n}^{T}\right) \boldsymbol{\omega}=P_{\mathbf{n}} \boldsymbol{\omega},
$$

where $P_{\mathbf{n}}=\mathbf{n n}^{T}$ is the projection matrix (components of the vectors are ordered into columns throughout this section).

Components of the unit normal vector $\mathbf{n}$ and the matrix $P_{\mathbf{n}}$ are expressed in the spherical coordinates as

$$
\mathbf{n}=\left(\begin{array}{c}
\sin \vartheta \cos \varphi \\
\sin \vartheta \sin \varphi \\
\cos \vartheta
\end{array}\right), \quad P_{\mathbf{n}}=\left(\begin{array}{ccc}
\sin ^{2} \vartheta \cos ^{2} \varphi & \sin ^{2} \vartheta \sin \varphi \cos \varphi & \sin \vartheta \cos \vartheta \cos \varphi \\
\sin ^{2} \vartheta \sin \varphi \cos \varphi & \sin ^{2} \vartheta \sin ^{2} \varphi & \sin \vartheta \cos \vartheta \sin \varphi \\
\sin \vartheta \cos \vartheta \cos \varphi & \sin \vartheta \cos \vartheta \sin \varphi & \cos ^{2} \vartheta
\end{array}\right)
$$

Thus, the projected vorticity vector $\boldsymbol{\omega}_{\mathbf{n}}=P_{\mathbf{n}} \boldsymbol{\omega}$ for $\boldsymbol{\omega}=\left(\omega_{x}, \omega_{y}, \omega_{z}\right)^{T}$ is expressed as

$$
\boldsymbol{\omega}_{\mathbf{n}}=\left(\begin{array}{c}
\omega_{x} \sin ^{2} \vartheta \cos ^{2} \varphi+\omega_{y} \sin ^{2} \vartheta \sin \varphi \cos \varphi+\omega_{z} \sin \vartheta \cos \vartheta \cos \varphi \\
\omega_{x} \sin ^{2} \vartheta \sin \varphi \cos \varphi+\omega_{y} \sin ^{2} \vartheta \sin ^{2} \varphi+\omega_{z} \sin \vartheta \cos \vartheta \sin \varphi \\
\omega_{x} \sin \vartheta \cos \vartheta \cos \varphi+\omega_{y} \sin \vartheta \cos \vartheta \sin \varphi+\omega_{z} \cos ^{2} \vartheta
\end{array}\right)
$$

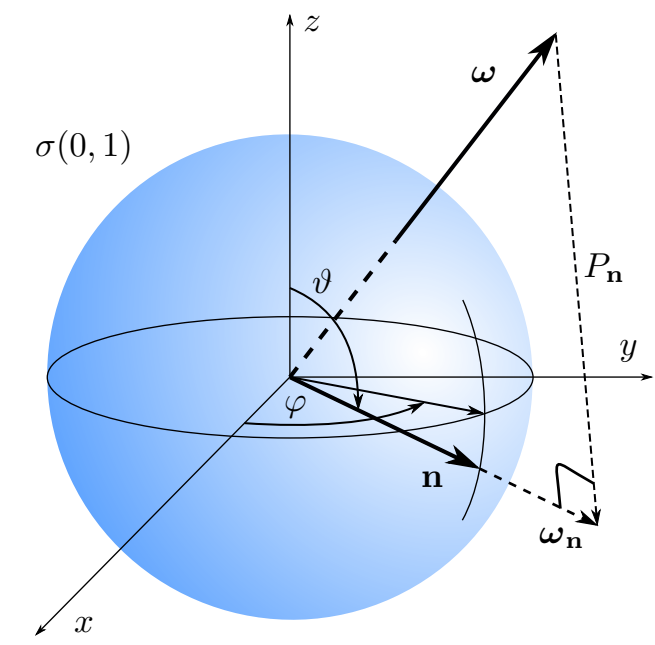

Figure 17: The projection of a vorticity vector according to (C.2).

Let us now integrate this quantity over all planes by averaging analogous to (15) to find the final averaged 
vorticity $\bar{\omega}$,

$$
\begin{aligned}
\overline{\boldsymbol{\omega}}\left(\mathbf{x}_{0}\right) & =\frac{\alpha}{4 \pi} \int_{0}^{2 \pi} \int_{0}^{\pi} \omega_{\mathbf{n}} \sin \vartheta \mathrm{d} \vartheta \mathrm{d} \varphi \\
& =\frac{\alpha}{4 \pi}\left(\begin{array}{c}
\int_{0}^{2 \pi} \int_{0}^{\pi}\left(\omega_{x} \sin ^{2} \vartheta \cos ^{2} \varphi+\omega_{y} \sin ^{2} \vartheta \sin \varphi \cos \varphi+\omega_{z} \sin \vartheta \cos \vartheta \cos \varphi\right) \sin \vartheta \mathrm{d} \vartheta \mathrm{d} \varphi \\
0 \\
\int_{0}^{2 \pi} \int_{0}^{\pi}\left(\omega_{x} \sin ^{2} \vartheta \sin \varphi \cos \varphi+\omega_{y} \sin \vartheta \sin \vartheta \sin ^{2} \varphi+\omega_{z} \sin \vartheta \cos \vartheta \sin \varphi\right) \sin \vartheta \mathrm{d} \vartheta \mathrm{d} \varphi \\
=
\end{array}\right)=\frac{\alpha}{4 \pi}\left(\begin{array}{c}
\omega_{x} \pi \int_{0}^{\pi} \sin ^{3} \vartheta \mathrm{d} \vartheta \\
\omega_{y} \pi \int_{0}^{\pi} \sin ^{3} \vartheta \mathrm{d} \vartheta \\
\omega_{z} 2 \pi \int_{0}^{\pi} \cos ^{2} \vartheta \sin \vartheta \mathrm{d} \vartheta
\end{array}\right)
\end{aligned}
$$

From our requirement that $\overline{\boldsymbol{\omega}}=\boldsymbol{\omega}$, we can immediately see that $\alpha=3$.

Another meaning of this derivation is worth noticing: by a component-wise integration of the projection matrix $P_{\mathbf{n}}$, we get

$$
\iint_{\sigma(0,1)} P_{\mathbf{n}} \mathrm{d} \sigma=\left(\begin{array}{ccc}
\frac{4}{3} \pi & 0 & 0 \\
0 & \frac{4}{3} \pi & 0 \\
0 & 0 & \frac{4}{3} \pi
\end{array}\right),
$$

i.e. the identity matrix scaled by $1 / 3$ of the surface of the sphere. Also note that the vorticity vector plays just a role of a constant vector in this derivation, and this scaling applies to any vector when the averaging (12) is applied to its projections of the type (C.2).

\section{References}

[1] Dallmann, U., "Topological structures of three-dimensional flow separation," Tech. Rep. 221-82 A07, Deutsche Forschungs- und Versuchsanstalt für Luft- und Raumfahrt DFVLR-IB, 1983.

[2] Vollmers, H., Kreplin, H.-P., and Meier, H., "Separation and vortical-type flow around a prolate spheroid - Evaluation of relevant parameters," Proceedings of the AGARD Symposium on Aerodynamics of Vortical Type Flows in Three Dimensions, Vol. AGARD-CP-342, Rotterdam, Netherlands, Apr. 1983, pp. 14-1-14-14.

[3] Hunt, J., Wray, A., and Moin, P., "Eddies, stream, and convergence zones in turbulent flows," Tech. rep., Center for Turbulence Research, 1988.

[4] Chong, M., "A general classification of three-dimensional flow fields," Physics of Fluids A, Vol. 2, No. 5, 1990, pp. $765-777$.

[5] Berdahl, C. and Thompson, D., "Eduction of swirling structure using the velocity gradient tensor," AIAA Journal, Vol. 31, No. 1, 1993, pp. 97-103.

[6] Jeong, J. and Hussain, F., "On the identification of a vortex," Journal of Fluid Mechanics, Vol. 285, 1995, pp. 69-94.

[7] Portela, L., Identification and characterization of vortices in the turbulent boundary layer, Ph.D. thesis, Stanford University, California, 1997.

[8] Cucitore, R., Quadrio, M., and Baron, A., "On the effectiveness and limitations of local criteria for the identification of a vortex," European Journal of Mechanics B/Fluids, Vol. 18, No. 2, 1999, pp. 261-282. 
[9] Zhou, J., Adrian, R., Balachandar, S., and Kendall, T., "Mechanisms for generating coherent packets of hairpin vortices in channel flow," Journal of Fluid Mechanics, Vol. 387, 1999, pp. 353-396.

[10] Sadarjoen, I. A. and Post, F. H., "Detection, quantification, and tracking of vortices using streamline geometry," Computers and Graphics, Vol. 24, 2000, pp. 333-341.

[11] Jiang, M., Machiraju, R., and Thompson, D., "A novel approach to vortex core region detection," Proceedings of the Joint Eurographics-IEEE TCVG Symposium on Visualization, Barcelona, Spain, May 2002, pp. 217-225.

[12] Chakraborty, P., Balachandar, S., and Adrian, R., "On the relationships between local vortex identification schemes," Journal of Fluid Mechanics, Vol. 535, 2005, pp. 189-214.

[13] Haller, G., "An objective definition of a vortex," Journal of Fluid Mechanics, Vol. 525, 2005, pp. 1-26.

[14] Zhang, S. and Choudhury, D., "Eigen helicity density: A new vortex identification scheme and its application in accelerated inhomogeneous flows," Physics of Fluids, Vol. 18, No. 5, 2006, pp. 058104$1-058104-4$.

[15] Kolár, V., "Vortex identification: New requirements and limitations," International Journal of Heat and Fluid Flow, Vol. 28, No. 4, 2007, pp. 638-652.

[16] Levy, Y., Degani, D., and Seginer, A., "Graphical visualization of vortical flows by means of helicity," AIAA Journal, Vol. 28, No. 8, 1990, pp. 1347-1352.

[17] Banks, D. and Singer, B., "A predictor-corrector technique for visualizing unsteady flow," IEEE Transactions on Visualization and Computer Graphics, Vol. 1, No. 2, 1995, pp. 151-163.

[18] Sujudi, D. and Haimes, R., "Identification of swirling flow in 3-D vector fields," AIAA Paper 95-1715, 12th AIAA CFD Conference, San Diego, CA, June 1995.

[19] Kida, S. and Miura, H., "Identification and analysis of vortical structures," European Journal of Mechanics B/Fluids, Vol. 17, No. 4, 1998, pp. 471-488.

[20] Roth, M. and Peikert, R., "A higher-order method for finding vortex core lines," Proceedings of IEEE Visualization '98, Research Triangle Park, NC, Oct. 1998, pp. 143-150.

[21] Strawn, R., Kenwright, D., and Ahmad, J., "Computer visualization of vortex wake systems," AIAA Journal, Vol. 37, No. 4, 1999, pp. 511-512.

[22] Roth, M., Automatic extraction of vortex core lines and other line-type features for scientific visualization, Ph.D. thesis, ETH, Zurich, Switzerland, 2000.

[23] Schafhitzel, T., Vollrath, J., Gois, J., Weiskopf, D., Castelo, A., and Ertl, T., "Topology-preserving $\lambda_{2}$-based vortex core line detection for flow visualization," Computer Graphics Forum, Vol. 27, No. 3, 2008, pp. 1023-1030.

[24] Stegmaier, S., Rist, U., and Ertl, T., "Opening the can of worms: An exploration tool for vortical flows," Proceedings of IEEE Visualization '05, Minneapolis, MN, Oct. 2005, pp. 463-470.

[25] Sahner, J., Weinkauf, T., and Hege, H.-C., "Galilean Invariant Extraction and Iconic Representation of Vortex Core Lines," Proceedings of the Joint Eurographics-IEEE VGTC Symposium on Visualization (Euro Vis 05), Leeds, UK, June 1-3, 2005, edited by K. W. Brodlie, D. Duke, and K. I. Joy, 2005, pp. 151-160.

[26] Wu, J.-Z., Xiong, A.-K., and Yang, Y.-T., "Axial stretching and vortex definition," Physics of Fluids, Vol. 17, No. 3, 2005, pp. 038108-1-038108-4.

[27] Maciel, Y., Robitaille, M., and Rahgozar, S., "A method for characterizing cross-sections of vortices in turbulent flows," International Journal of Heat and Fluid Flow, Vol. 37, 2012, pp. 177-188. 
[28] Baysal, K. and Rist, U., "Identification and quantification of shear layer influences on the generation of vortex structures," New Results in Numerical and Experimental Fluid Mechanics VII, edited by A. Dillmann, G. Heller, W. Nitsche, and H.-P. Kreplin, Springer, 2010, pp. 241-248.

[29] Koláŕ, V., Moses, P., and Šístek, J., "Triple Decomposition Method for Vortex Identification in TwoDimensional and Three-Dimensional Flows," Computational Fluid Dynamics 2010, Proceedings of 6th ICCFD Conference, St. Petersburg, Russia, July 12-16, 2010, edited by A. Kuzmin, Springer, 2011, pp. $225-231$.

[30] Kolár, V., Moses, P., and Š́stek, J., "Local corotation of line segments and vortex identification," Proceedings of the 17th Australasian Fluid Mechanics Conference, Paper 110, edited by G. Mallinson and J. Cater, Australasian Fluid Mechanics Society, 2010, 4 pages.

[31] Bažant, Z. and Oh, B., "Efficient numerical integration on the surface of a sphere," ZAMM Zeitschrift für Angewandte Mathematik und Mechanik, Vol. 66, No. 1, 1986, pp. 37-40.

[32] Hannay, J. and Nye, J., "Fibonacci numerical integration on a sphere," Journal of Physics A: Mathematical and General, Vol. 37, No. 48, 2004, pp. 11591-11601.

[33] Ehret, A., Itskov, M., and Schmid, H., "Numerical integration on the sphere and its effect on the material symmetry of constitutive equations-A comparative study," International Journal for Numerical Methods in Engineering, Vol. 81, 2010, pp. 189-206.

[34] Bake, S., Meyer, D., and Rist, U., "Turbulence Mechanism in Klebanoff Transition: a Quantitative Comparison of Experiment and Direct Numerical Simulation," Journal of Fluid Mechanics, Vol. 459, 2002, pp. 217-243.

[35] Šístek, J., Kolář, V., Cirak, F., and Moses, P., "Fluid-Structure Interaction and Vortex Identification," Proceedings of the 18th Australasian Fluid Mechanics Conference, Paper 125, edited by P. A. Brandner and B. W. Pearce, Australasian Fluid Mechanics Society, 2012, 4 pages.

[36] Chakraborty, P., Balachandar, S., and Adrian, R. J., "Comment on "Axial stretching and vortex definition" [Phys. Fluids 17, 038108 (2005)]," Physics of Fluids, Vol. 18, No. 2, 2006, pp. 029101-1-029101-2.

[37] Wu, J.-Z., Xiong, A.-K., and Yang, Y.-T., "Response to "Comment on "Axial stretching and vortex definition"' [Phys. Fluids 18, 029101 (2006)]," Physics of Fluids, Vol. 18, No. 2, 2006, pp. 029102-1$029102-2$.

[38] Pierce, B., Moin, P., and Sayadi, T., "Application of vortex identification schemes to direct numerical simulation data of a transitional boundary layer," Physics of Fluids, Vol. 25, No. 1, 2013, pp. 015102$1-015102-14$.

[39] Koláŕ, V., "Compressibility effect in vortex identification," AIAA Journal, Vol. 47, No. 2, 2009, pp. 473475.

[40] Jiang, M., Machiraju, R., and Thompson, D., "Detection and visualization of vortices," The Visualization Handbook, edited by C. Hansen and C. Johnson, Elsevier, 2005, pp. 295-309. 